\title{
Phonon eigenfunctions of inhomogeneous lattices: Can you hear the shape of a cone?
}

\author{
Grace H. Zhang and David R. Nelson \\ Department of Physics, Harvard University, Cambridge, MA 02138, USA.
}

(Dated: July 27, 2021)

\begin{abstract}
We study the phonon modes of interacting particles on the surface of a truncated cone resting on a plane subject to gravity, inspired by recent colloidal experiments. We derive the ground state configuration of the particles under gravitational pressure in the small cone angle limit, and find an inhomogeneous triangular lattice with spatially varying density but robust local order. The inhomogeneity has striking effects on the normal modes such that an important feature of the cone geometry, namely its apex angle, can be extracted from the lattice excitations. The shape of the cone leads to energy crossings at long wavelengths and frequency-dependent quasi-localization at short wavelengths. We analytically derive the localization domain boundaries of the phonons in the limit of small cone angle and check our results with numerical results for eigenfunctions.
\end{abstract}

\section{INTRODUCTION}

"Can you hear the shape of a drum?" is a classic problem formulated by Mark Kac: Can we determine the geometry of a two-dimensional membrane from the eigenvalues of the Laplace equation [1]? This seminal work led to a proliferation of studies on extracting geometrical information from a system's normal modes. For example, Ref. [2] examines how the geometry of the pore-grain interface in a porous media can be recovered from the eigenvalues and eigenfunctions of the diffusion propagator. These works have focused on the membrane as a continuous medium. In this paper, we study the effect of a discrete periodic lattice structure with a slowly varying lattice constant on problems of this category. We focus on truncated cones, and as in particular whether the fundamental shape parameter of the cone angle can be inferred from the normal modes.

When interacting particles ordered in a two-dimensional plane are under inhomogeneous strains (e.g. due to a gravitational pressure), they can in general remove the extra energy cost, a form of geometrical frustration, in at least two distinct ways: by forming topological defects in an otherwise homogeneous lattice, or by maintaining a defect-free lattice by curving into the third dimension [3]. We study here the latter scenario. As we shall see, the overall shape of the curved membrane is coupled with lattice spacings that can generally be non-uniform, converting the lattice into an inhomogeneous crystal.

Inhomogeneous crystals are collections of particles with nonuniform equilibrium densities but robust well-ordered local structures. Experimentally, such lattices have been observed in plasmas under gravitational or flow fields [6] 8], foams between curved surfaces [9, ions in laser traps [10, and, most recently, colloidal particles confined by an applied electric field [11. However, a complete understanding of inhomogeneous crystals remains elusive, because the inhomogeneity of the lattice seems incompatible with ideas from condensed matter physics that typically assume perfect periodicity.

In this paper, we study a two-dimensional (2d) inhomogeneous crystal, consisting of interacting particles that deform from a cylindrical shell into a truncated cone under gravitational hydrostatic pressure. In the spirit of inverse problems, can we infer information on the shape of this cone (such as the inclination angle) from its normal modes (in this case, the in-plane phonons of an inhomogeneous lattice)? We answer in the affirmative. Slow adiabatic changes in the lat- tice spacing, controlled by the cone angle, manifest at long wavelengths as a reordering of low energy eigenmodes, and at short wavelengths as quasi-localization due to a spatially varying local band edge, the maximum energy (or frequency) beyond which lattice phonons cannot propagate. Our results could be checked experimentally, for example, on systems of overdamped density-mismatched colloidal particles adsorbed onto conical surfaces [12, 13.

The paper is organized as follows. In Sec. III as a simplified warm up problem, we illustrate inhomogeneityinduced quasi-localization of lattice excitations using a onedimensional (1d) model of dislocation pileups [14, 15], which exhibits the same phenomenon as the $2 \mathrm{~d}$ conic sheet studied in the main body of the paper. In Sec. III] we argue that a truncated cone with a slowly varying lattice constant is the ground state of interacting particles with cylindrical boundary conditions under gravitational pressure. In Sec. [V], we study the effect of inhomogeneity on the in-plane phonons. We find that low frequency phonons exhibit interesting energy crossings as a function of the cone angle (Sec. IV B) and high frequency phonons are localized inside spatial domains whose sizes depend on the cone angle. We derive the localization domain boundaries as a function of the phonon frequency and the angle of the cone, using two complementary methods: We recover this boundary (1) in momentum space by identifying the spatially-varying band edge (Sec. IV C), and (2) in real space by a mapping to Schrodinger's equation and applying an inverted Wentzel-Kramers-Brillouin (WKB) analysis (or the Liouville-Green method, also due to Jeffreys) [16 20] (Sec. IV D].

\section{ONE-DIMENSIONAL EXAMPLE: DISLOCATION PILEUPS}

We expect that the localization effect we find for inhomogeneous cones (Sections III and IV) occurs more generally in inhomogeneous lattices. Here, we illustrate this phenomenon in a relatively simple one-dimensional inhomogeneous lattice with long range interactions and only longitudinal phonons, inspired by the physics of dislocation pileups [14, 15. Specifically, we show that the normal modes of $1 \mathrm{~d}$ dislocation pileups, a type of defect assembly embedded in two-dimensional crystals [14, exhibit quasi-localization as a function of the phonon frequency, due to a position-dependent band edge. Readers are referred to Appendix A for details and may skip ahead to Sec. III without loss of continuity. 
Dislocation pileups exemplify an intriguing class of $1 \mathrm{~d}$ inhomogeneous lattices, whose constituents are not particles but point-like edge dislocation defects in a two-dimensional host crystal 14. The dislocations, once emitted in response to a stress field, all have the same Burgers vector topological charge and interact via a long-ranged logarithmic potential, with an average dislocation density determined by the form of the external shear stress [15].

Fig. 1 shows the eigenfunctions for two types of pileups, resulting from applied stress fields in the two-dimensional host lattice that are uniform (Fig. 1 a) and linearly varying (Fig. 1b) in space. The eigenfunctions are stacked vertically according to their eigenenergies $\Omega$. For both types of pileup, the eigenfunctions become more localized as $\Omega$ increases, with a localization domain concentrated within the densest parts of the pileup as the eigenmode frequency increases.

We find the localization domain boundaries of the longitudinal pileup phonon modes by calculating the band edge of the phonon dispersion for a uniform pileup (see Appendix A or Ref. [15]), given by the simple condition,

$$
\Omega_{\text {edge }}=\frac{Y b^{2} \pi}{16 D^{2}}
$$

where $D$ is the average dislocation spacing of the pileup, $Y$ is the Young's modulus of the $2 \mathrm{~d}$ host crystal, and $b$ is the magnitude of the dislocation Burger's vector. Eigenmodes with frequencies above this threshold $\Omega>\Omega_{\text {edge }}$ enter the band gap and decay exponentially, since the dislocation lattice cannot resolve and propagate the phonon, instead generating strong back scattering (Bragg diffraction in one dimension). When the dislocation lattice spacing is spacedependent $D=D(x)$, as in the case for the pileups shown in Fig. 1. the local band edge becomes slowly varying in space, with $\Omega=\Omega(x)$. On using Eq. (1), the localization domain $\left[x^{-}, x^{+}\right]$and energy $\Omega$ of the band edge satisfies,

$$
\Omega\left(x^{ \pm}\right)=\frac{Y b^{2} \pi}{16} n\left(x^{ \pm}\right)^{2} .
$$

where $n(x)$ is the average dislocation charge density, related to the dislocation lattice spacing via $D(x)=|n(x)|^{-1}$, given by

$$
\begin{aligned}
n_{\mathrm{D}}(x) & =\frac{4 \sigma_{0}}{Y b} \frac{x}{\sqrt{\left(\frac{L}{2}\right)^{2}-x^{2}}} \\
n_{\mathrm{SC}}(x) & =\frac{4 \sigma_{0}}{Y b} \sqrt{1-\left(\frac{x}{L / 2}\right)^{2}}
\end{aligned}
$$

for the eigenfunctions shown in Fig. 1, and $Y$ is the Young's modulus of the $2 \mathrm{~d}$ crystal, $b$ is the magnitude of the dislocation Burgers vector, and $\sigma_{0}$ is the strength of the applied shear stress. Note that the domain of localization tracks the square of the dislocation density profile $\sim n(x)^{2}$. Eq. (2) is plotted in red Fig. 1 and shows good agreement with the localization domains of our numerical eigenfunctions for the two dislocation densities shown.

Nonlinear interactions between the dislocation degrees of freedom is essential for the quasi-localization phenomena. In contrast, a chain of balls and springs with constant stiffness on an inclined surface, which also assumes an inhomogeneous configuration, does not exhibit quasi-localization because the interaction strengths embodied in the spring constants are independent of the inhomogeneous lattice spacing (see Appendix B for details).

(a)
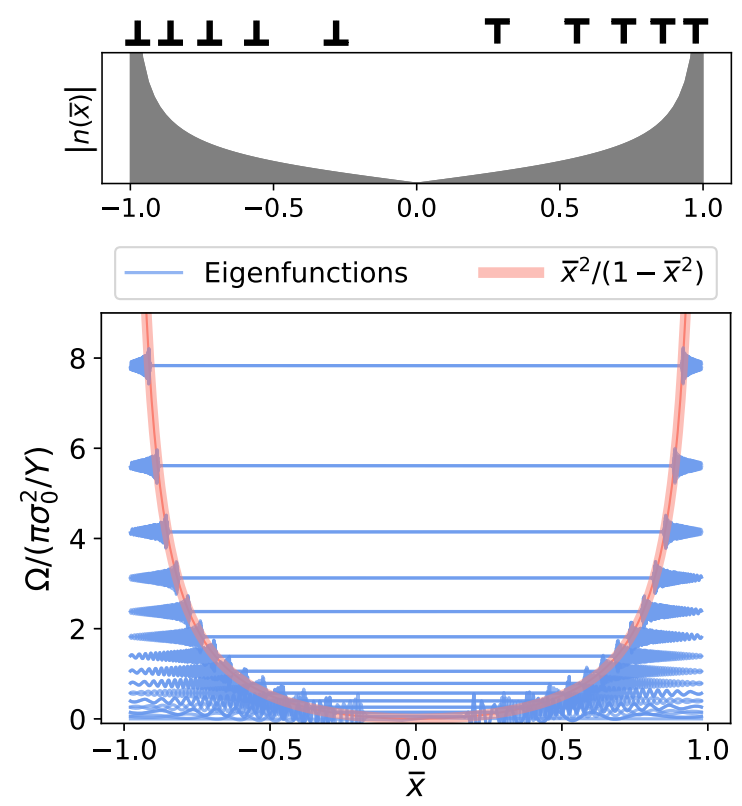

(b)
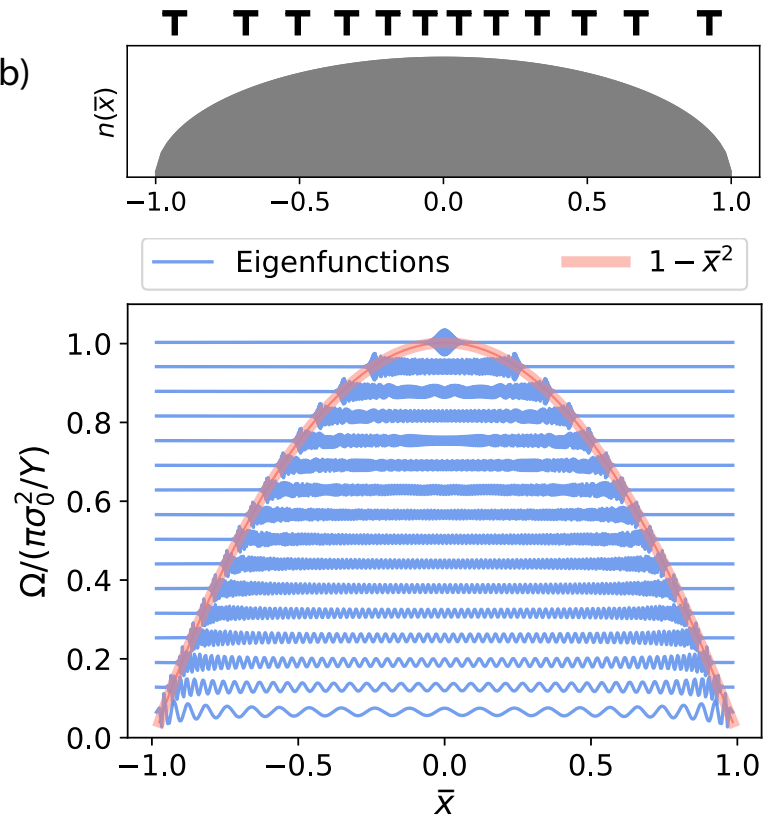

FIG. 1. The localization boundaries in one dimension predicted by Eq. 22 (red lines) and the numerical phonon eigenfunctions (blue) for two types of pileups with different average dislocation densities $n(\bar{x})$, where $\bar{x} \equiv x /(L / 2)$, plotted in gray and illustrated by schematics of dislocations. The eigenfunctions are stacked vertically according to their rescaled eigenenergies $\Omega$. As predicted by Eq. (2), the localization boundaries are proportional to $n(x)^{2}$ : the eigenfunctions localize towards the densest parts of the dislocation lattice (the edges in (a) and the center in (b)) as $\Omega$ increases.

\section{CONE SHAPE}

In this section, we introduce a particularly simple twodimensional microscopic model with an inhomogeneous lat- 
tice constant: particles initially within a $2 \mathrm{~d}$ cylindrical sheet interacting via a Lennard-Jones (LJ) pair potential, which then deforms under gravitational pressure (Sec. III A below). Unlike a sheet in the flat plane with gravity pointing downwards in the plane of the sheet (see Appendix C), a cylindrical shell subjected to a weak gravitational field parallel to the cylinder axis relieves its frustration by transforming into a truncated cone with an inhomogeneous lattice spacing (see Sec. III B. In Sec. III C, we map our discrete particle model onto continuum elasticity theory to derive the cone angle as a function of the microscopic parameters and the spatially varying lattice spacing as a function of the cone surface coordinates. (In Sec. IV] we will show that information about the cone angle can also be inferred from the phonon spectrum.)

\section{A. Microscopic model}

Consider a $2 \mathrm{~d}$ system of particles interacting via a pair potential $V_{\text {int }}$ and experiencing an external one-body potential $V_{\text {ext }}$ :

$$
H\left[\left\{\vec{r}_{n}\right\}\right]=\sum_{n \neq m} V_{\mathrm{int}}\left(\vec{r}_{n}, \vec{r}_{m}\right)+\sum_{n=1}^{N} V_{\mathrm{ext}}\left(\vec{r}_{n}\right)
$$

where $N$ is the total number of particles and $n, m$ are particle indices. For concreteness, we work with an isotropic interaction potential $V_{\text {int }}\left(\vec{r}, \vec{r}^{\prime}\right)=V_{\text {int }}\left(\left|\vec{r}-\vec{r}^{\prime}\right|\right)$ of the Lennard-Jones form,

$$
V_{\text {int }}(r)=\varepsilon\left[\left(\frac{a_{0}}{r}\right)^{12}-2\left(\frac{a_{0}}{r}\right)^{6}\right],
$$

which has both a repulsive and an attractive component, with a potential minimum at $r=a_{0}$. (Upon setting $\sigma \equiv$ $2^{-1 / 6} a_{0}$, Eq. 6 can be rewritten in the usual way, $V_{\text {int }}(r)=$ $4 \varepsilon\left[\left(\frac{\sigma}{r}\right)^{12}-\left(\frac{\sigma}{r}\right)^{6}\right]$.) Without an external potential, particles in the ground state form a triangular lattice with a uniform lattice constant $a_{0}$ (see Fig. 2a) [21]. However, as shown in the next section, the classical ground state can be distorted in an interesting way by a nonzero gravitational potential,

$$
V_{\text {ext }}(\vec{r})=m g z
$$

where $m$ is the effective particle mass and $g$ is the gravitational acceleration. In colloidal experiments, $m$ can be changed by tuning the density mismatch between the colloid and the solvent. The pair potential in Eq. 5 is assumed to act in three dimensions, along a chord connecting atoms embedded in a cone or cylinder. In settings where the colloids are adsorbed onto a cylindrical or conical template, or the bending rigidity is very large, flexural phonons are frozen out. Henceforth, we ignore flexural phonons and focus only on the physics of in-plane fluctuations in Sec. IV.

The microscopic model in Eq. (5)- (7) can be mapped at long wavelengths onto continuum elasticity theory. We use the latter tool to calculate the inhomogeneous equilibrium lattice positions under the gravitational potential in the next section, and elaborate on the details of this mapping in Sec. IIIC.

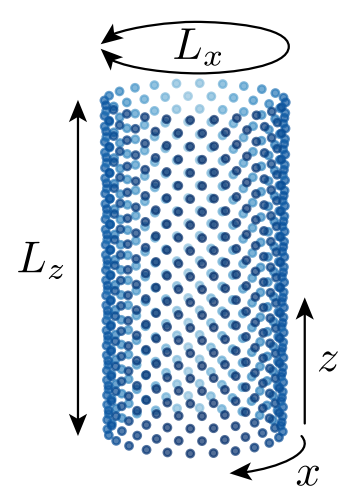

(a)

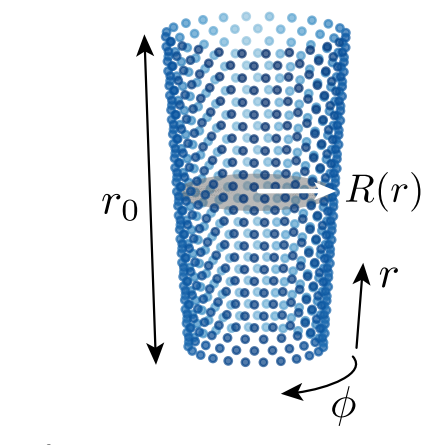

(b)

(c)
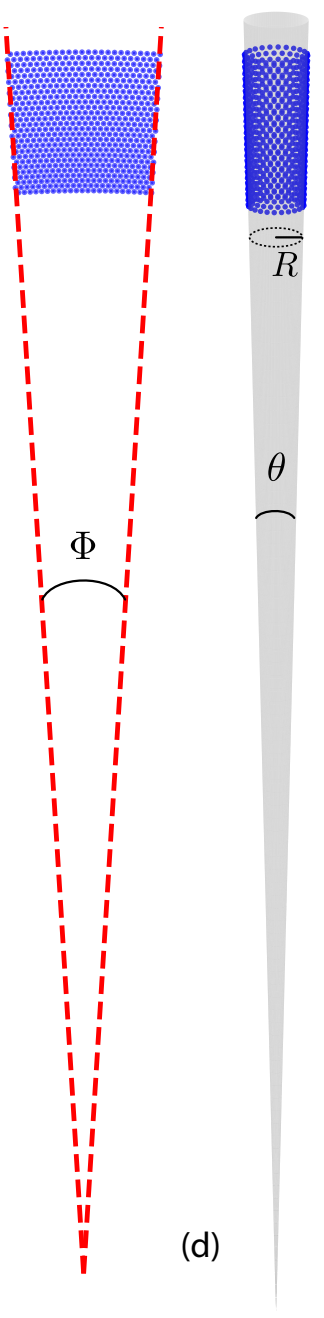

FIG. 2. (a): The ground state configuration of $N$ particles interacting via the Lennard-Jones potential in a cylindrical sheet is a triangular lattice with lattice constant $a_{0}$, height $L_{z}$, and rim circumference $L_{x}$. (b): Under gravitational pressure, the cylinder deforms into a truncated cone with length $r_{0}$. The coordinates of the cone are the azimuthal angle $\phi$, the longitudinal length along the surface $r$. The cross-sectional radius $R=R(r)$ increases as a function of $r$. (d)-(e): The sector angle $\Phi$ of the rolled out cone (d) is related to the apex angle $\theta$ (e) by $\theta=\Phi / \pi$. The numerical parameters used in this figure are $\left(L_{z}, L_{x}, a_{0}, N, \theta\right)=\left(13 \sqrt{3}, 24,1,648,1.1^{\circ}\right)$.

\section{B. Elasticity theory}

We first examine a $2 \mathrm{~d}$ cylindrical sheet with uniform elastic coupling constants using continuum elasticity theory and then calculate the equilibrium displacements under gravitational pressure. As detailed in Appendix E these displacements hold for the lattice of interacting particles in Eq. (5) when the gravitational pressure is sufficiently weak such that,

$$
\frac{\alpha_{0}}{2 B} \frac{L_{z}}{2} \ll 1
$$

where $B=\mu+\lambda$ is the bulk modulus of the unperturbed crystal. In this limit, the resulting configuration describes a truncated cone (Fig. 2b), with the precise correspondence shown in Eqs. 222-26 below. Thus, a cylinder whose cir- 
cular base is free to slide on the flat surface on which it rests, collapses downward and inward under gravitational pressure, resulting in the cylinder base being smaller and denser than its less deformed upper end.

A two-dimensional cylindrical sheet, with periodic boundary conditions imposed along $x$ and the axis of symmetry pointing along $z$, under a gravitational potential can be studied as for the similar problem of a $3 \mathrm{~d}$ elastic solid subject to gravity, as discussed in Ref. [22]. Under an isotropic pressure that increases with decreasing vertical coordinate $z$ (lower portions of the sheet bear more weight from above), the cylindrical sheet's free energy is given by

$$
F=\frac{1}{2} \int d^{2} r\left(2 \mu u_{i k}^{2}+\lambda u_{i i}^{2}+2 \alpha_{0}\left(L_{z}-z\right) u_{i i}\right),
$$

where $\delta p=\alpha_{0}(L-z)$ is the isotropic pressure, $u_{i j}=$ $\frac{1}{2}\left(\frac{\partial u_{i}}{\partial r_{j}}+\frac{\partial u_{j}}{\partial r_{i}}\right)$ is the strain tensor, $\mu$ and $\lambda$ are the first and second $2 \mathrm{~d}$ Lamé coefficients. Here $\alpha_{0}$ is proportional to the gravitational constant $g$ controlling the hydrostatic pressure of, say, density-mismatched colloids in a solvent (see Eq. (34) below) and $\vec{u}(\vec{r})$ is the displacement of the sheet from its $\alpha_{0}=0$ equilibrium configuration at position $\vec{r}$. The nondeformed $\left(\alpha_{0}=0\right)$ cylindrical sheet has a height of $L_{z}$ and a rim circumference of $L_{x}$. The natural boundary conditions for this problem are (1) anchoring the bottom edge of the sheet at the base, which requires that the vertical displacements $u_{z}$ vanish at $z=0$, and (2) the stresses vanish at the upper edge $z=L_{z}$ of the truncated cone.

In the absence of pressure $\left(\alpha_{0}=0\right)$, the free energy in Eq. (9) is minimized when the equilibrium displacements $\bar{u}_{i}$ vanish and the strain tensor $\bar{u}_{i j}=0$ for all $i, j$. We now calculate the equilibrium positions under nonzero $\alpha_{0}$ by finding the set of uniform strains $\bar{u}_{i j}$ that minimize Eq. $(9)$. Since the gravitational term is isotropic and does not depend on $u_{z x}$, we immediately have $\bar{u}_{z x}=0$. Upon setting the derivatives of the free energy $F$ with respect to $u_{z z}$ and $u_{x x}$ to zero and using the boundary conditions $\bar{u}_{z z}=\bar{u}_{x x}=0$ at $z=L_{z}$, we obtain the polar projection of the displacements as (see Fig. 2p and Appendix C for details),

$$
\begin{aligned}
& \bar{u}_{z}=\frac{\alpha_{0}}{2 B}\left(\frac{z^{2}}{2}-L_{z} z-\frac{x^{2}}{2}\right) \\
& \bar{u}_{x}=\frac{\alpha_{0}}{2 B}\left(z-L_{z}\right) x .
\end{aligned}
$$

where $B=\mu+\lambda$ is the $2 \mathrm{~d}$ bulk modulus. Upon assuming the gravitational pressure to be sufficiently weak (Eq. (8)) and imposing periodic boundary conditions by wrapping the sheet around in the $x$ direction to form a cone, we obtain the axial and azimuthal displacements in terms of the surface coordinates as,

$$
\begin{aligned}
& \bar{u}_{r}=\frac{\alpha_{0}}{2 B}\left(\frac{r^{2}}{2}-r_{0} r\right) \\
& \bar{u}_{\phi}=\frac{\alpha_{0}}{2 B} \frac{L_{x}}{2 \pi}\left(r-r_{0}\right) \phi,
\end{aligned}
$$

where $r$ is the longitudinal coordinate along the surface, $r_{0}$ is the longitudinal length of the truncated cone, $\phi$ is the azimuthal angle around the cone axis (see Fig. 2p), and the zero displacement boundary condition at the bottom edge $\bar{u}_{r}=0$ at $r=0$ is now satisfied, in contrast to Eq. 110.

Upon redefining $u_{i} \rightarrow u_{i}-\bar{u}_{i}$ in Eq. (9), where $\bar{u}_{i}$ is the equilibrium displacement of the cone relative to the cylinder, we can eliminate (gauge away) the pressure term in the free energy (see Appendix F1 for details) and obtain, up to an additive constant,

$$
F=\frac{1}{2} \int d^{2} r\left(2 \mu u_{i k}^{2}+\lambda u_{i i}^{2}\right)
$$

We now calculate the apex angle $\theta$ of the cone (Fig. 2 $\mathrm{d}$ ), as a function of the gravitational pressure coefficient $\alpha_{0}$ and elastic constants $\mu$ and $\lambda$, from the sector angle of an unfolded cone given by (see Fig. 2k),

$$
\tan \frac{\Phi}{2}=\frac{\Delta x}{\Delta z}
$$

where,

$$
\begin{aligned}
& \Delta x=\bar{u}_{x}\left(x=\frac{L_{x}}{2}, z=L_{z}\right)-\bar{u}_{x}\left(x=\frac{L_{x}}{2}, z=0\right) \\
& \Delta z=\bar{u}_{z}\left(x=\frac{L_{x}}{2}, z=L_{z}\right)-\bar{u}_{z}\left(x=\frac{L_{x}}{2}, z=0\right)+L_{z} .
\end{aligned}
$$

Upon substituting Eqs. (10) and (11) into Eq. (16), we obtain, as a function of $\alpha_{0}$, the cone dimensions, and the bulk modulus $B=\mu+\lambda$,

$$
\begin{aligned}
\Delta x & =\frac{\alpha_{0}}{2 B} \frac{L_{x} L_{z}}{2} \\
\Delta z & =-\frac{\alpha_{0}}{2 B} \frac{L_{z}^{2}}{2}+L_{z} .
\end{aligned}
$$

The sector angle is then given by, using Eq. 15,

$$
\tan \frac{\Phi}{2}=\frac{\frac{\alpha_{0}}{2 B} L_{x}}{2\left(1-\frac{\alpha_{0}}{2 B} \frac{L_{z}}{2}\right)} .
$$

If the gravitational pressure is weak enough such that Eq. (8) holds, we can approximate $\Phi$ to first order in $\alpha_{0}$ as,

$$
\Phi \approx \frac{\alpha_{0}}{2 B} L_{x}+O\left[\left(\frac{\alpha_{0}}{2 B} \frac{L_{z}}{2}\right)^{2}\right]
$$

plotted as red dashed lines in Fig. 26. The apex angle (Fig. 2d) in this weak pressure regime is then given by $\theta=\Phi / \pi$ as,

$$
\theta \approx \frac{\alpha_{0}}{2 \pi B} L_{x}
$$

We now determine the smoothly varying lattice spacing $a(z)$ produced by this cone angle, using Eq. (11), as,

$$
\begin{aligned}
a(z) & =a_{0}+\bar{u}_{x}\left(x=a_{0}, z\right)-\bar{u}_{x}(x=0, z) \\
& =a_{0}\left[1+\frac{\alpha_{0}}{2 B}\left(z-L_{z}\right)\right]
\end{aligned}
$$

where $a_{0}$ is the lattice constant of the truncated cone at its upper edge, i.e. height $z=L_{z}$. The vertical coordinate $z$ of the unrolled flat sheet is proportional to the longitudinal coordinate $r$ climbing up along the surface of the truncated cone (see Fig. 2p). When the gravitational pressure is weak enough such that the approximation in Eq. (20) holds, we can rewrite Eq. 23 as,

$$
a(r)=a_{0}\left[1+\theta \frac{\pi}{L_{x}}\left(r-r_{0}\right)\right]
$$


where $r_{0}$ is the total length of the cone along its inclined surface, as indicated in Fig. 2b, and we have written $r_{0}=$ $L_{z}\left(1-\theta \frac{\pi}{2} \frac{L_{z}}{L_{x}}\right) \approx L_{z}$, an approximation accurate to first order in $\theta=\Phi / \pi$.

The spatially varying lattice spacing in Eq. (24), derived using the elasticity solution in Eq. (11), is precisely that of a cylindrical lattice deformed isotropically onto the surface of a cone with apex angle $\theta$, given by,

$$
\begin{aligned}
\frac{a(r)}{a_{0}} & =\frac{R(r)}{R_{0}}=\frac{r \sin (\theta / 2)}{L_{x} / 2 \pi} \\
& \approx 1+\theta \frac{\pi}{L_{x}}\left(r-r_{0}\right)
\end{aligned}
$$

where $R \equiv r \sin \frac{\theta}{2}$ is the radius of the horizontal cross section of the cone (see Fig. $2 \mathrm{~d}$ ), and $R_{0}$ is the cross-sectional radius at the top of the truncated cone. Finally, Eqs. (11) and (24) show that the lattice constants at the top of the cone are not distorted, since the gravitational pressure $-2 \alpha_{0}\left(L_{z}-z\right) u_{i i}$ vanishes there in our model, as is reasonable because there are no particles above this top rim exerting gravitation forces. Hence, the circumference $L_{x}$ of the cylindrical rim is equal to the circumference of the top end of the truncated cone.

Finally, we note that in arriving at the solutions above, we have assumed constant effective coupling coefficients $\mu$ and $\lambda$. In the case of interaction potentials $V_{\text {int }}(r)$ which depend on the interparticle distance, inhomogeneity in the ground state lattice would render the Lamé coefficients space-dependent. This nonlinear feedback would manifest in Eq. (9) as,

$$
F=\frac{1}{2} \int d^{2} r\left(2 \mu(\vec{r}) u_{i j}^{2}+\lambda(\vec{r}) u_{i i}^{2}+2 \alpha_{0}\left(L_{z}-z\right) u_{i i}\right)
$$

However, we show in Appendix E that this nonlinearity can be neglected in the small angle regime (i.e. Eq. (8)), where the ground state equilibrium displacements are wellapproximated by those provided by the linear solutions in Eqs. 10 and (11). However, as the numerical calculations in Sec.IV show, this inhomogeneity in the elastic coefficients have a non-negligible effect on the normal modes and is key to the physics of fluctuations.

\section{Connection between elasticity theory and a microscopic model}

We now further detail the mapping between the microscopic model in Sec. IIIA and the elastic free energy in Sec. IIIB. In the following sections, we express the pressure coefficient $\alpha_{0}$ and elastic constants $\mu, \lambda$ in Eq. (9), and the cone angle $\theta$ in Eq. 21, in terms of the parameters of the specific microscopic Hamiltonian Eq. (5), using a LennardJones pair potential.

\section{Gravitational pressure}

Upon integrating the gravitational part of the continuum free energy Eq. (9) by parts, we obtain,

$$
\begin{aligned}
F_{\mathrm{ext}} & =\alpha_{0} \int d^{2} r\left(L_{z}-z\right) u_{i i} \\
& =-\alpha_{0} \int d^{2} r\left[\partial_{i}\left(L_{z}-z\right)\right] u_{i}(\vec{r})+S
\end{aligned}
$$

The surface term $S$ vanishes, as can be seen by evaluating

$$
\begin{aligned}
S= & \alpha_{0}\left[\left.\int d z\left(L_{z}-z\right) u_{x}\right|_{x=0} ^{x=L_{x}}+\left.\int d x\left(L_{z}-z\right) u_{z}\right|_{z=0} ^{z=L_{z}}\right](30) \\
= & \alpha_{0}\left\{\int\left(L_{z}-z\right)\left[u_{x}\left(x=L_{x}, z\right)-u_{x}(x=0, z)\right] d z\right. \\
& \left.+L_{x}\left[\left(L_{z}-L_{z}\right) u_{z}\left(x, z=L_{z}\right)-\left(L_{z}-0\right) u_{z}(x, z=0)\right]\right\}
\end{aligned}
$$

The first term vanishes by our cylindrical boundary conditions along $x$ and the last term vanishes by the fixed boundary condition at $z=0$. The free energy due to the external potential then becomes,

$$
\begin{aligned}
F_{\text {ext }} & =\alpha_{0} \int d^{2} r \delta_{i z} u_{i}(\vec{r}) \\
& =\alpha_{0} a^{2} \sum_{n} z_{n}+\text { const. }
\end{aligned}
$$

Upon identifying,

$$
\alpha_{0}=\frac{m g}{a^{2}},
$$

where $g$ is the gravitational acceleration and $m$ is the effective mass of one particle, we see that $F_{\text {ext }}$ corresponds to $H_{\text {ext }}$ from the microscopic Hamiltonian in Eq. (7), up to an additive contribution to the energy.

\section{Effective Lame coefficients}

To find the effective elastic coefficients corresponding to the microscopic Hamiltonian such as Eq. (5), we first recall that the dispersion relations at long wavelengths for a homogeneous lattice with short range interactions is given by elasticity theory as [21,

$$
\begin{aligned}
& \Omega_{T}=m \omega_{T}^{2}=\mu(q a)^{2}, \\
& \Omega_{L}=m \omega_{L}^{2}=(2 \mu+\lambda)(q a)^{2}
\end{aligned}
$$

where $q$ is the momentum, $\omega_{L}$ and $\omega_{T}$ are the longitudinal and transverse phonon frequencies, respectively, and $\Omega_{L}$ and $\Omega_{T}$ are the corresponding eigenenergies. As shown later in Sec. IV C (see also Appendix F 2), for particles in a triangular lattice interacting via the Lennard-Jones potential in Eq. (6), the dispersion curves at long wavelengths are,

$$
\begin{aligned}
& m \omega_{T}^{2}=(q a)^{2} \varepsilon \frac{27}{a^{2}} \\
& m \omega_{L}^{2}=(q a)^{2} \varepsilon \frac{3 \cdot 27}{a^{2}} .
\end{aligned}
$$

Thus, the effective Lamé coefficients for our microscopic Lennard-Jones model are given by,

$$
\mu=\lambda=27 \frac{\varepsilon}{a^{2}}
$$

We immediately see that the elastic constants are spatially varying if the lattice spacing $a=a(r)$ is spatially varying, as in the case of the cone. Note that the corresponding Poisson ratio $\nu=\lambda /(2 \mu+\lambda)$ is equal to $1 / 3$, which satisfies the Cauchy condition for isotropic interaction potentials [23]. (We note that for systems of particles with sufficiently longrange repulsive interactions such as $V_{\text {int }}(r) \sim 1 / r$, the effective $\lambda$ becomes infinite in the long wavelength limit [24, 25]. 
This is equivalent to the observation that the Wigner crystal is incompressible [24.)

\section{Cone Angle}

Upon combining Eqs. 20, 34, and 39, we obtain the apex angle of the cone in terms of the microscopic Lennard-Jones pair potential parameter $\varepsilon$ as,

$$
\theta=\frac{L_{x} m g}{108 \pi \varepsilon}
$$

Thus, under a gravitational field parallel to the axis of symmetry, a cylindrical shell of circumference $L_{x}$, consisting of particles with effective mass $m$ interacting via the LJ potential in Eq. (6) with strength $\varepsilon$, will assume the shape of a cone with angle $\theta$ given by Eq. 40.

\section{CONE PHONONS: FREQUENCY-DEPENDENT LOCALIZATION}

Can you hear the shape of a cone? In this section, we examine the eigenmodes of a $2 d$ lattice in the shape of a truncated cone. We find that the cone shape, namely its apex angle $\theta$, controls level crossings at long wavelengths and eigenfunction quasi-localization at short wavelengths.

In Sec. IV A, we study the fluctuation energy to second order in particle displacements. In Sec. IVB, we show that the low frequency normal modes have robust vortex and anti-vortex configurations in their eigenfunctions, and exhibit changes in energy ordering as a function of the cone angle $\theta$.

In the upper half of the phonon spectrum, we find that eigenmodes at high frequencies are confined towards the bottom of the cone, where the lattice is most compressed. We use two methods to identify the analytical relation between the eigenenergy $\Omega$ and the position $r^{*}$ of the localization domain boundary, measured from the base up the flanks of the cone. In Sec. IV C, we find the space-dependent local band edge (the maximum eigenenergy above which all modes decay exponentially) by calculating the dispersion relation. In Sec. IVD we map our equations of motion onto Schrodinger's equation and find the turning point using an upside-down WKB analysis. These two methods give identical results: the localization domains $\left[0, r^{*}\right]$ of the highfrequency eigenfunctions on a cone with small apex angle $\theta$ are given, in terms of the parameters $\varepsilon$ and $a_{0}$ specifying the Lennard-Jones pair potential (Eq. (6)), by,

$$
r^{*}(\Omega ; \theta)=r_{0}+\frac{3}{31 \pi} \frac{L_{x}}{\theta}\left[C\left(\varepsilon, a_{0}\right)-\ln \Omega\right]
$$

where $r_{0}$ is the total length along the cone flank, $C\left(\varepsilon, a_{0}\right)=$ $432 \varepsilon / a_{0}^{2}$ is a constant, and $L_{x}$ and $a_{0}$ are the circumference and lattice constant, respectively, of the undeformed cylinder. (The parameter $a_{0}$ is also the lattice constant along the top rim of the truncated cone where the lattice is undeformed by the gravitational field.) Thus, given the phonon eigenenergy $\Omega$, Eq. (41) predicts the location $r^{*}$ above which its eigenfunction decay exponentially. Note that $r$ and $r^{*}$ in Eq. (41) can be replaced with $z$ and $z^{*}$ to first order in $\theta$. Finally, Eq. 41, evaluated when $r^{*}=r_{0}$ also gives the threshold eigenfrequency $\Omega_{\text {deloc }}$ below which all eigenfunctions are delocalized,

$$
\ln \Omega_{\text {deloc }}=C\left(\varepsilon, a_{0}\right) .
$$

The criterion embodied in Eq. 41) is plotted in green in Fig. 3 and shows good agreement with the numerical eigenfunctions.

\section{A. Phonon spectrum}

To examine the fluctuations about the conical ground state in Figs. 2 $\mathrm{p}$ and c, we decompose the position of the $n$-th particle as $\vec{r}_{n}=\vec{R}_{n}+\vec{u}_{n}$, where $\vec{R}_{n}$ is its equilibrium position and $\vec{u}_{n}$ is its displacement away from equilibrium. Hence forth, $i, j, k=1,2$ will be used as direction indices and $n, m=1, \cdots N$ as particle indices.

Upon expanding the fluctuation energy to second order in displacements $\vec{u}_{n}$, the linear terms vanish by force balance. The gravitational potential, although it determines the spatial variation of the lattice constant in the ground state, drops out. On neglecting constant terms, we obtain the fluctuation energy as,

$$
\Delta E=\sum_{n \neq m} \frac{1}{2} \Pi_{i j}\left(\vec{R}_{n}-\vec{R}_{m}\right)\left(u_{n}-u_{m}\right)_{i}\left(u_{n}-u_{m}\right)_{j}
$$

where, for the Lennard-Jones interaction potential in Eq. (6), $\Pi_{i j}(\Delta \vec{R})$ is given by,

$$
\begin{aligned}
\Pi_{i j}(\Delta \vec{R})=\varepsilon & {\left[a_{0}^{12}\left(12(12+2) \frac{\Delta R_{i} \Delta R_{j}}{|\Delta \vec{R}|^{12+4}}-12 \frac{\delta_{i j}}{|\Delta \vec{R}|^{12+2}}\right)\right.} \\
& \left.-2 a_{0}^{6}\left(6(6+2) \frac{\Delta R_{i} \Delta R_{j}}{|\Delta \vec{R}|^{6+4}}-6 \frac{\delta_{i j}}{|\Delta \vec{R}|^{6+2}}\right)\right]
\end{aligned}
$$

As a check, we will compare our analytical results in Sec. IV C and IV D against numerical eigenfunctions. These eigenfunctions are obtained by solving the real-space eigenproblem,

$$
\mathbf{M u} \mathbf{u}^{(\alpha)}=\Omega_{\alpha} \mathbf{u}^{(\alpha)}
$$

with fixed boundary conditions at the lower rim, where $\alpha$ indicates the $\alpha$-th normal mode and the dynamical matrix $\mathbf{M}$ depends on $\Pi_{i j}$ (see Appendix F 3 for details).

\section{B. Properties of low frequency eigenmodes}

The low frequency normal modes exhibit interesting behavior as a function of the apex angle $\theta$. Fig. 4 shows the the five lowest eigenenergies and their eigenfunctions at $\theta=0$ (the limit of the cylinder) and $\theta \approx 2.42^{\circ}$. We can classify the eigenfunctions by node-counting. The blue lines mark where the horizontal displacement $u_{x}$ crosses 0 and the red lines mark where the vertical displacement $u_{z}$ crosses 0 . Note that the crossing of two longitudinal node lines of different orientations (i.e. a horizontal blue $\left(u_{x}=0\right)$ line and a vertical red $\left(u_{z}=0\right)$ line) coincides with a vortex-like texture in the displacement field, while the crossing of two transverse node lines of difference orientations (i.e. a vertical blue 

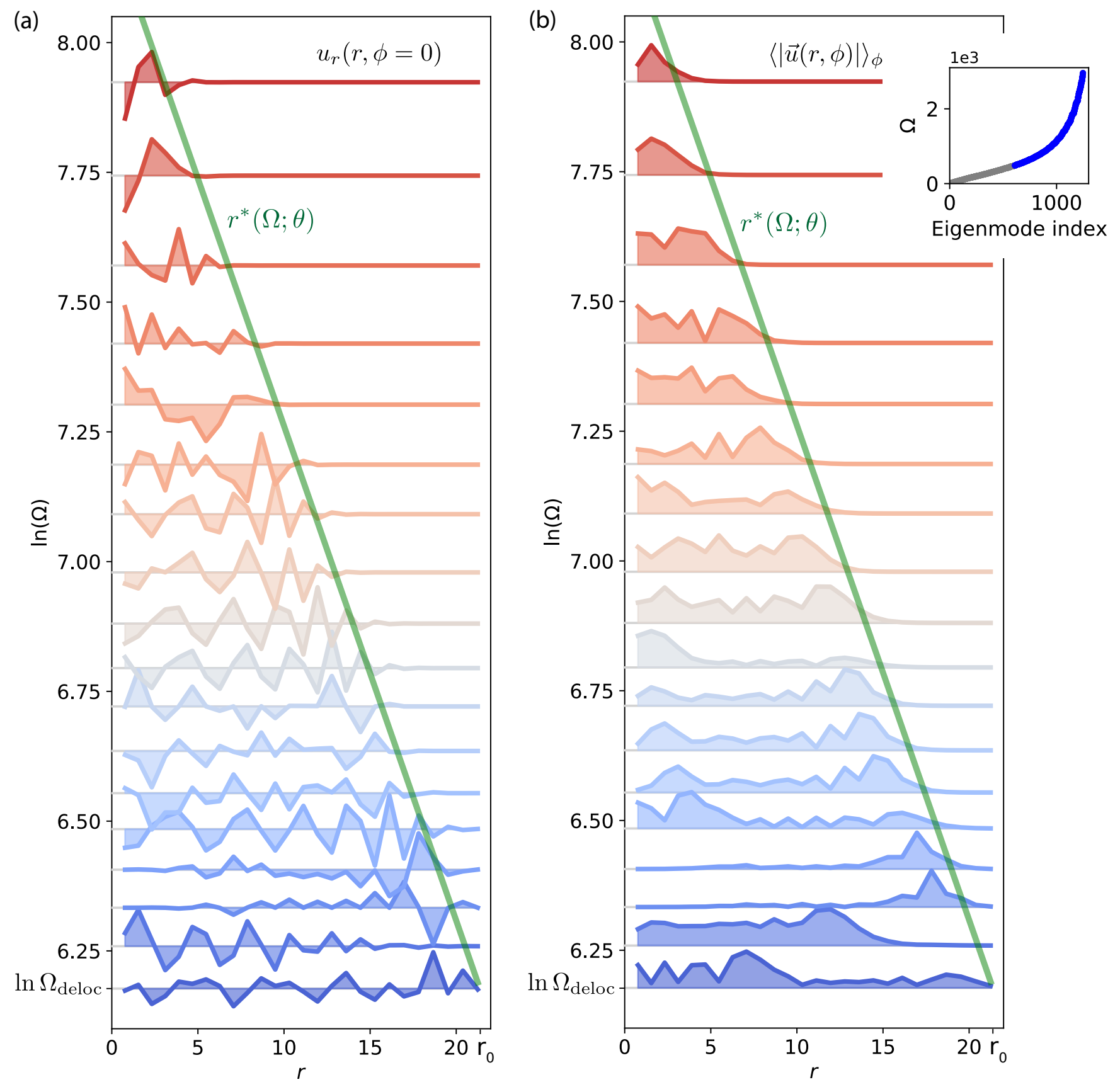

FIG. 3. Localization of high frequency eigenfunctions for $N$ particles interacting via a LJ potential on the surface of a cone, with geometrical parameters $\left(L_{z}, L_{x}, N, \theta\right)=\left(13 \sqrt{3}, 24,648,1.1^{\circ}\right)$, longitudinal cone length $r_{0} \approx 21.25$, and undeformed lattice constant $a_{0}=1$. (a)-(b): The longitudinal component (a) and absolute magnitude (b) of the numerical eigenfunctions stacked up vertically according to the logarithm of their eigenenergies. In (a), $u_{r}(r, \phi=0)$ is the longitudinal displacement at one value of $\phi$ and $\langle|\vec{u}(r, \phi)|\rangle_{\phi}$ is the displacement magnitudes in each row of the cone averaged over the azimuthal angle $\theta$ (e.g. for lattice sites with the same $r$ coordinate). The green line is Eq. (41), which clearly identifies the localization domain boundaries of the eigenmodes as a function of $\Omega$. $\Omega_{\text {deloc }}$ marks the eigenenergy below which all eigenfunctions are delocalized. The inset on the right plots the eigenenergies $\Omega$ as a function of the eigenmode index. The blue points highlight the range of eigenenergies whose corresponding eigenmodes experience quasi-localization.

$\left(u_{x}=0\right)$ line and a horizontal red $\left(u_{z}=0\right)$ line) corresponds to an anti-vortex configuration in the displacement field. These vortices and anti-vortices in the eigenfunctions are stable to perturbations, or in other words, topologically protected. These vortex configurations are thus preserved as we tune the apex angle away from 0 . In contrast, the crossing between a transverse node line and a longitudinal node line (i.e. two red lines or two blue lines) are not robust and 


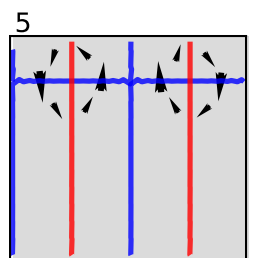

3

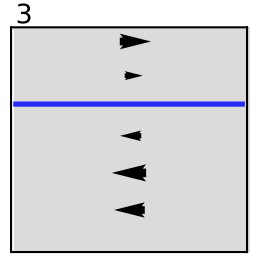

1

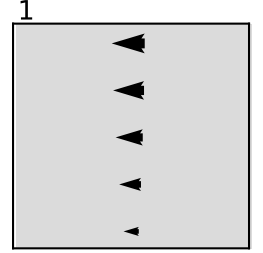

$$
\theta=0^{\circ}
$$

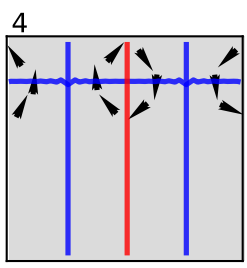

2

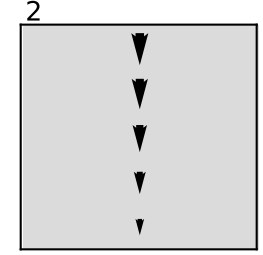

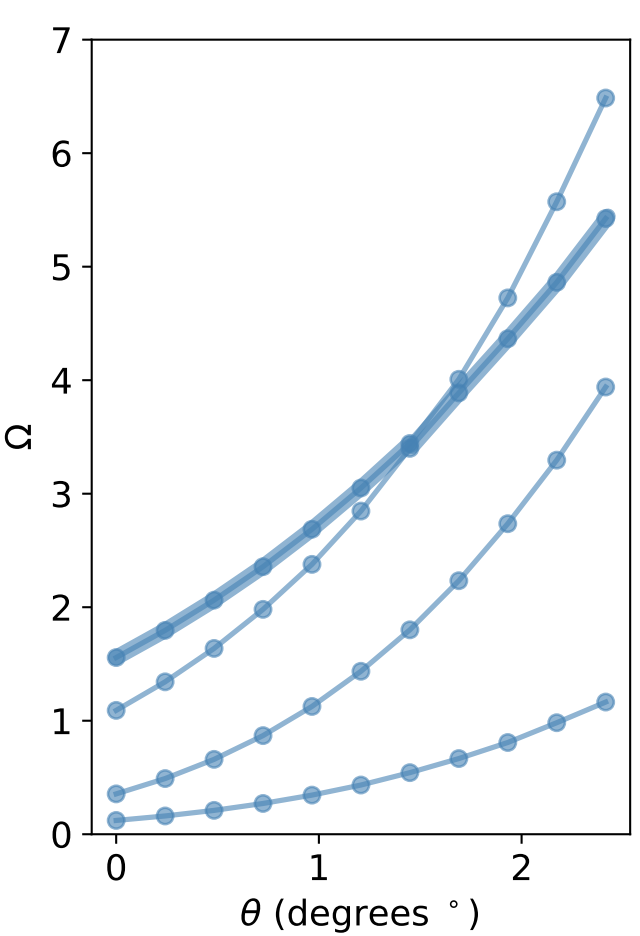

5

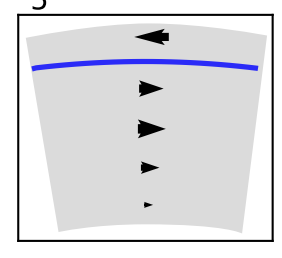

3

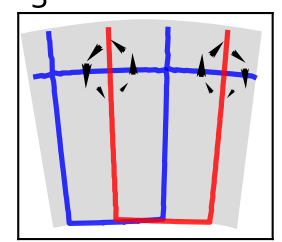

1

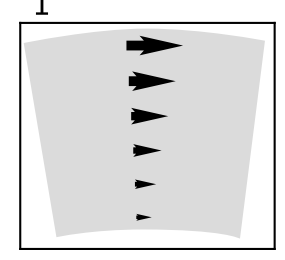

$$
\theta=2.4^{\circ}
$$

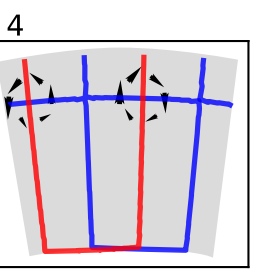

2

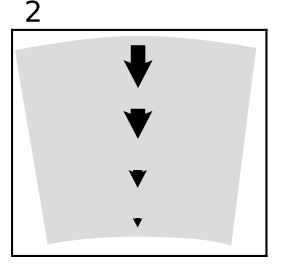

FIG. 4. The central plot shows the five lowest energy levels of the cone as a function of increasing apex angle $\theta$. The corresponding eigenmodes are shown in the cylindrical limit $\theta=0^{\circ}$ (left) and at $\theta=2.4^{\circ}$ (right). The enlarged black arrows indicate the displacements at selected lattice sites. The blue and red lines indicate zeros of the horizontal displacements $u_{x}$ and vertical displacements $u_{z}$, respectively. The level crossing between the ( $\left.\mathrm{T}, n_{r}=1, n_{\phi}=0\right)$ mode and the $\left(\mathrm{T}, n_{r}=1, n_{\phi}=1\right)$, ( $\left.\mathrm{T}, n_{r}=1, n_{\phi}=-1\right)$ doublet (shown as the thick line in the central plot) occurs at $\theta \approx 1.5^{\circ}$. As discussed in the text $T$ specifies a transverse long wavelength mode, while $n_{r}$ and $n_{\phi}$ are integers specifying node numbers.

can easily vanish under a slight conical deformation.

Since the vortex configurations stay robust under the conical perturbation, we can use them to identify and track the eigenmodes as we tune $\theta$ adiabatically. For a cylinder $(\theta=0)$, the number of nodes along the $x$ and $z$ directions, $n_{x}$ and $n_{z}$, determine the wave numbers of the eigenfunctions at long wavelengths. For periodic boundary conditions along $x$ and bottom clamped boundary condition along $z$, these wavefunctions behave according to,

$$
u(x, z) \sim \sin \left(k_{z} z\right) \cos \left(k_{x} x\right)
$$

where,

$$
\begin{array}{rlr}
k_{z}=\frac{\pi}{L_{z}}\left(n_{z}+\frac{1}{2}\right), & n_{z}=0,1,2, \cdots \\
k_{x}=\frac{2 \pi}{L_{x}} n_{x}, & n_{x}=0, \pm 1, \pm 2, \cdots
\end{array}
$$

Note that in cone coordinates, we have $n_{z} \rightarrow n_{r}$ and $n_{z} \rightarrow$ $n_{\phi}$. Additionally, since we are in two dimensions, the eigenmodes at low energies split into two branches, transverse and longitudinal (see Fig. 5). To combine this information, we can classify each low energy normal mode by identifying whether it is transverse $(\mathrm{T})$ or longitudinal $(\mathrm{L})$, and then counting the number of nodes along $r\left(n_{r}\right)$ and $\phi\left(n_{\phi}\right)$. For example, in the cylindrical limit $\theta=0$, the eigenmodes, in order of increasing frequency, are: $\left(\mathrm{T}, n_{r}=0, n_{\phi}=0\right),(\mathrm{L}$, $\left.n_{r}=0, n_{\phi}=0\right),\left(\mathrm{T}, n_{r}=1, n_{\phi}=0\right),\left(\mathrm{T}, n_{r}=1, n_{\phi}=1\right)$, $\left(\mathrm{T}, n_{r}=1, n_{\phi}=-1\right)$.
In contrast, at $\theta=2.42^{\circ}$, this ordering changes to: $(\mathrm{T}$, $\left.n_{r}=0, n_{\phi}=0\right),\left(\mathrm{L}, n_{r}=0, n_{\phi}=0\right),\left(\mathrm{T}, n_{r}=1, n_{\phi}=1\right),(\mathrm{T}$, $\left.n_{r}=1, n_{\phi}=-1\right)$, (T, $\left.n_{r}=1, n_{\phi}=0\right)$. That is, eigenmode 3 swaps order with eigenmodes 4 and 5 . As shown in Fig. 4 this level crossing occurs at approximately $\theta \approx 1.52^{\circ}$. Note that eigenmodes 4 and 5 at $\theta=0$ (eigenmodes 3 and 4 after the level crossing) are degenerate doublets.

This level crossing is interesting for two reasons. First, it suggests that we can probe the angle of the cone by measuring the ordering of its lowest energy eigenmodes. Second, we know that for Hermitian matrices, level crossings for a system with $k$ parameters can only occur on a $(k-2)$ manifold 26 28]. Since our conical system has only one parameter $(\theta)$, level crossings are not allowed unless some symmetry is present to relax the condition. What accidental symmetry in this system allows the eigenvalues to cross when $\theta \approx 1.5^{\circ}$, is an interesting question for future investigations.

\section{Moving band edges}

In this section, we derive the localization domain boundary of the high frequency phonons on the cone by first calculating the band edge of the dispersion relations for the interacting particles on a uniform triangular lattice. Provided the lattice parameter varies slowly in space (i.e. if the external gravitational field is weak), we can compute a local band edge that varies spatially throughout the cone as a function of $r$, giving us a stopping criterion for a fixed phonon eigenenergy 
$\Omega$. We compare the predicted domain boundary to numerical eigenfunctions and see good agreement.

Since the Lennard-Jones (LJ) interaction potential is short ranged, we truncate to nearest neighbor interactions in the following calculations. (As detailed in Appendix F 2, the addition of next nearest neighbor interactions has a negligible effect on the band edge.) Upon rewriting the summation in Eq. 43 as a sum over center sites $\left\{\vec{R}_{m}\right\}$ and their nearest neighbors $\left\{\vec{R}_{m}+\vec{n}_{\alpha} \mid \alpha=1, \cdots, 6\right\}$, where $\left\{\vec{n}_{\alpha}\right\}$ are the 6 nearest neighbor lattice vectors, we obtain,

$$
\begin{aligned}
\Delta E & =\frac{1}{2} \sum_{m} \sum_{i j} \sum_{\alpha=1, \cdots, 6} \Pi_{i j}\left(\vec{n}_{\alpha}\right)\left(u_{i}\left(\vec{R}_{m}+\vec{n}_{\alpha}\right)-u_{i}\left(\vec{R}_{m}\right)\right) \\
& \times\left(u_{j}\left(\vec{R}_{m}+\vec{n}_{\alpha}\right)-u_{j}\left(\vec{R}_{m}\right)\right)
\end{aligned}
$$

After incorporating the dynamical term $\sum_{n} \frac{m}{2}\left|\dot{\vec{u}}_{n}\right|^{2}$ in the Hamiltonian, calculating the equations of motion, and assuming a wave-like solution of the form,

$$
u_{i}\left(\vec{R}_{m}\right)=e^{i \vec{q} \cdot \vec{R}_{m}} e^{i \omega t} u_{i}(\vec{q})
$$

where $\vec{q}$ is the momentum and $\omega$ is the physical frequency of the in-plane phonons, we arrive at the following eigenvalue problem,

$$
\Omega(\vec{q}) u_{i}(\vec{q})=D_{i j}(\vec{q}) u_{j}(\vec{q})
$$

where the dynamical matrix $D_{i j}(\vec{q})$ is given by,

$$
D_{i j}(\vec{q})=\sum_{\alpha=1, \cdots, 6} \Pi_{i j}\left(\vec{n}_{\alpha}\right)\left(1-\cos \left(\vec{n}_{\alpha} \cdot \vec{q}\right)\right)
$$

and $\Omega(\vec{q})=m \omega(\vec{q})^{2}$ are its eigenvalues.

Calculation of the dynamical matrix and its eigenvalues is detailed in Appendix F 2. Fig. 5 shows the analytical dispersion curves in the first Brillouin zone. Note that, interestingly, upon traversing a single band in a continuous loop in momentum space $\Gamma-K-M-\Gamma$, starting from and circling back the zone origin $\Gamma$, a transverse branch (orange) transforms smoothly into a longitudinal branch (blue) upon returning to $\Gamma$, and vice versa.

As shown in Appendix F2, the upper band edge of the spectrum (i.e. the symmetry point $M$ in Fig. 5 for a uniform lattice with an LJ pair potential and lattice constant $a_{0}$ is simply,

$$
\Omega_{\text {edge }}=432 \frac{\varepsilon}{a_{0}^{2}} .
$$

For a slowly varying inhomogeneous lattice with $a=a(r)$, we expect that the band edge becomes space-dependent as,

$$
\Omega_{\text {edge }}[a(r)]=\frac{6 \varepsilon}{a(r)^{2}}\left[152\left(\frac{a_{0}}{a(r)}\right)^{12}-80\left(\frac{a_{0}}{a(r)}\right)^{6}\right]
$$

Upon substituting Eq. (24) for the lattice spacing $a(r)$ of the cone into the band edge criterion in Eq. (53) and expanding to first order in $\theta$, we recover the localization condition for small cone angles displayed in Eq. 41.

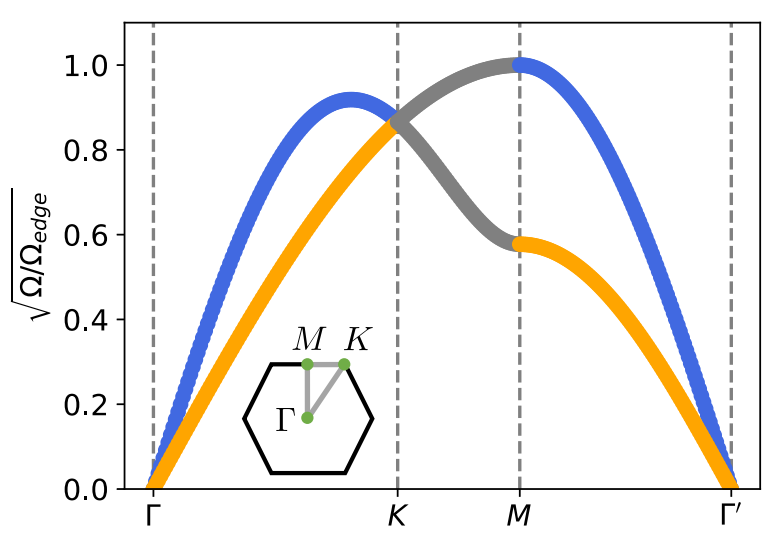

FIG. 5. Phonon dispersion curves for a uniform triangular lattice resulting from nearest neighbor interactions under the LennardJones potential along the $\Gamma-K-M-\Gamma^{\prime}$ loop of the first Brillouin zone, as shown in the inset. Near the Brillouin zone center $\Gamma$, lower phonon branches have transverse characteristics (orange) and higher phonon branches have longitudinal characteristics (blue). High frequency modes along the Brillouin zone boundary have mixed transverse and longitudinal properties (gray). Note that as one moves from left to right along the blue-grayorange path on the circuit above, a longitudinal phonon excitation smoothly changes into a transverse mode. A similar transformation takes place along the orange-gray-blue path from $\Gamma$ to $\Gamma^{\prime}$.

\section{Effective potential and Inverted WKB}

In this section, we obtain further insights by identifying a change of variable that smooths out the rapidly varying displacement field of the phonon at the band edge. This transformation allows us to map our eigenvalue equation in the continuum limit onto a Schrodinger-like equation with the momentum transformation $p \rightarrow i p$. We then apply an inverted 1d WKB analysis to the cone along the $r$ direction to arrive at an alternative derivation of Eq. (41).

Figure 6a shows the highest frequency eigenmode for the cylinder. The displacement field varies rapidly in space on the scale of the lattice constant, and gradually vanishes near the top and bottom edges. In Fig. 7月, we subtract the gradual modulation in the $z$ direction to resolve the shortwavelength pattern of the displacements. By applying a change of variables that flips the sign of the displacement in every other row and every other column (marked by the colored sites in Fig. 7 a), we arrive at a configuration where the displacements in every other row are approximately identical in orientation and magnitude, while the rows alternate between pure vertical displacement and pure horizontal displacements (Fig. 7b).

Upon applying this change of variables to the band-edge eigenfunction in Fig. 6a, the transformed displacement field in Fig. 6p has two key characteristics: (1) displacements within the same row are identical, and (2) displacements in every other row vary smoothly along the vertical direction. In Fig. $7 \mathrm{p}$, the red (blue) stripes highlights the sites aligned in the horizontal direction, interleaved by rows where there is zero vertical (horizontal) displacement.

Hence, we reduce the two-dimensional eigenfunction problem to a one-dimensional one along the $z$ coordinate of the 
(a)

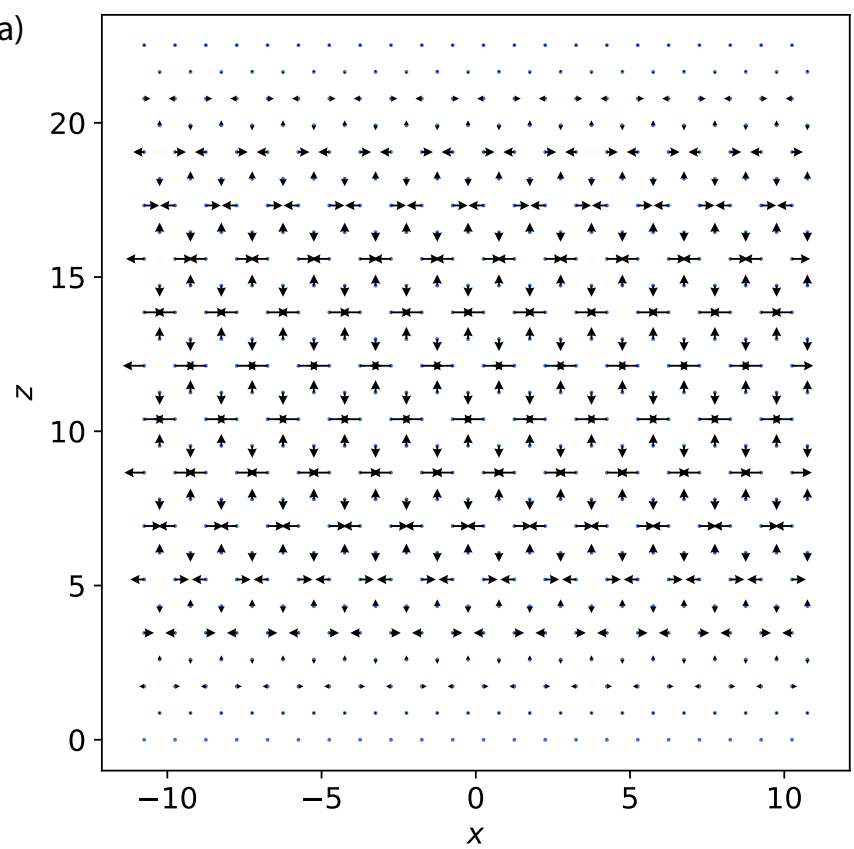

(b)

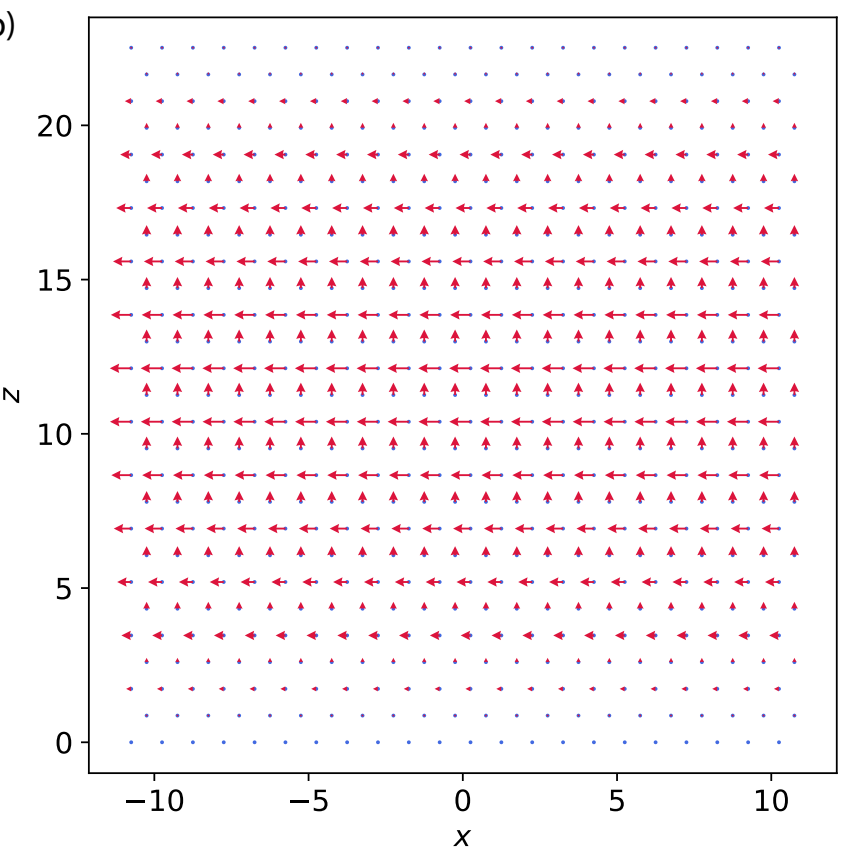

FIG. 6. The displacements corresponding to the highest energy eigenmode of the cylinder with a free boundary at the top and a fixed boundary at the bottom before (a) and after (b) the change of variables illustrated in Fig. 7 .

cylinder (i.e. the $r$ coordinate along the flank of the cone), as indicated by the vertical stripes in Fig. 7 b. We then "integrate out" the intercepting row, where the displacement direction abruptly rotates by $90^{\circ}$, by calculating an effective spring constant between two horizontally aligned sites. As shown in Fig. 7p, to calculate this effective spring constant, we imagine stretching the vertical distance between the two sites by $\Delta z$. We then calculate the elastic energy $\Delta E_{s}$ from

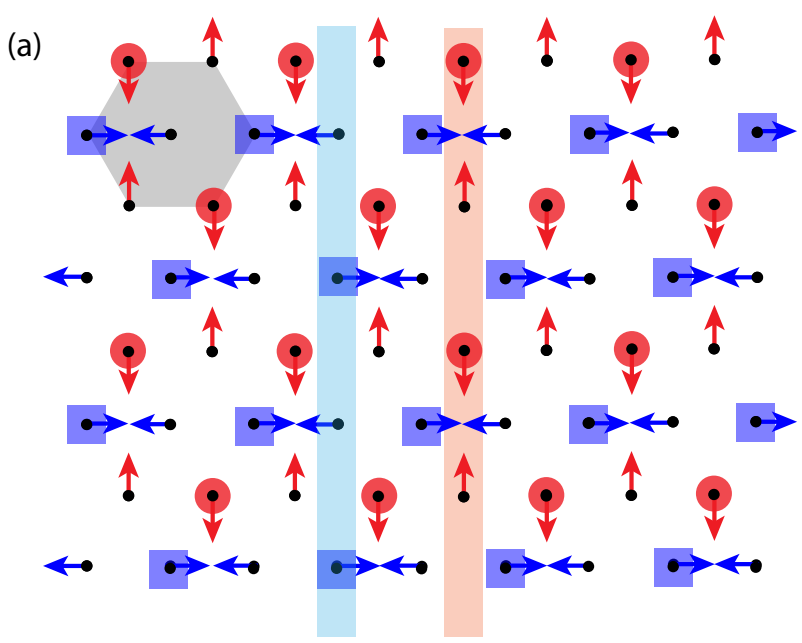

(b)

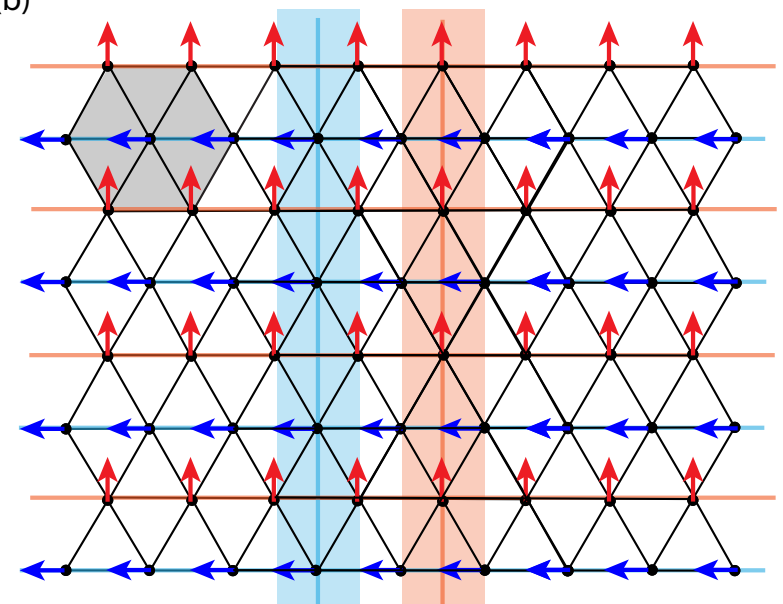

(c)

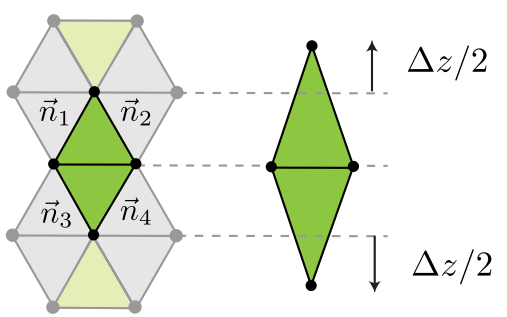

FIG. 7. (a): Displacement configuration of the highest frequency eigenmode for the cylindrical shell. The presence of a colored shape at a site indicates that the sign of the displacement there is flipped according to our change of variables. Red circle (blue square) indicates that the flipped displacement at that site is purely vertical (horizontal). Note that along each vertical stripe, the displacement at every other site is flipped. (b): The red (blue) vertical stripe highlights the sites aligned in the horizontal direction, interleaved by rows where there are zero vertical (horizontal) displacements. We coarse grain over each interleaving row by extracting an effective spring constant. (c): Vertical stretching of a pair of sites aligned in the horizontal direction by $\Delta z$. The effective spring constant $k_{1 \mathrm{~d}}$ is extracted from the elongation energy of the four affected bonds $\left\{\vec{n}_{b} \mid b=1, \cdots, 4\right\}$.

this stretching $\Delta u_{i}= \pm \delta_{i 2} \Delta z / 2$ using Eq. 44 as follows,

$$
\Delta E_{s}(\Delta z)=\sum_{b=1, \cdots, 4} \Pi_{i j}\left(\vec{n}_{b}\right)\left(\delta_{2 i} \frac{\Delta z}{2}\right)\left(\delta_{2 j} \frac{\Delta z}{2}\right),
$$


where $\left\{\vec{n}_{b}\right\}$ consists of the four bonds (labeled in Fig. 7k) whose lengths are elongated by the vertical stretching. Upon rewriting the elastic energy in the form of Hooke's law,

$$
\Delta E_{s}(\Delta z) \equiv \frac{1}{2} k_{1 \mathrm{~d}}(\Delta z)^{2}
$$

we extract the effective spring constant as,

$$
k_{1 \mathrm{~d}}(r)=\frac{3}{2} \frac{\varepsilon}{a(r)^{2}}\left[152\left(\frac{a_{0}}{a(r)}\right)^{12}-80\left(\frac{a_{0}}{a(r)}\right)^{6}\right] .
$$

where the spatially varying lattice constant $a=a(r)$ for the cone is given by Eq. (24).

We can now model the vertical stripe in Fig. $7 \mathrm{~b}$ as a $1 \mathrm{~d}$ chain of springs with the effective spring constant given in Eq. (56). The resulting Lagrangian is given by,

$$
\mathcal{L}[\mathbf{u}]=\sum_{n}\left[\frac{m}{2} \dot{u}_{n}^{2}-\frac{1}{2} k_{1 d}\left[a\left(r_{n}\right)\right]\left(u_{n}-u_{n+1}\right)^{2}\right],
$$

where $u_{n}$ is the displacement scalar of the $n$-th site along the vertical strip. We can choose to model the red or blue strip in Fig. 7, for which $u_{n}$ would represent the vertical or horizontal component of the displacement; these quantities have the same equations of motion.

As highlighted in Fig. 7a, the change of variables flips the displacement at every other site in this 1d chain: $u_{n-1}, u_{n+1} \rightarrow-u_{n-1},-u_{n+1}$. Upon applying this change of variables, the equations of motion simplify to,

$$
\left(-\Omega+4 k_{n}\right) u_{n}+k_{n}\left(u_{n+1}-2 u_{n}+u_{n-1}\right)=0,
$$

where $\Omega \equiv m \omega^{2}$ as before, $k_{n} \equiv k_{1 d}\left(r_{n}\right)$, and we have approximated $k_{n} \approx\left(k_{n}+k_{n-1}\right) / 2$. Upon taking the continuum limit by setting $\partial_{r}^{2} u\left(r_{n}\right)=\left(u_{n-1}-2 u_{n}+u_{n+1}\right) / a_{n}^{2}$, where $a_{n}=a\left(r_{n}\right)$, we arrive at the following equation of motion in the continuum limit,

$$
\frac{1}{2 m_{\mathrm{eff}}(r)} \partial_{r}^{2} u(r)+V_{\mathrm{eff}}(r) u(r)=\Omega u(r),
$$

where,

$$
V_{\mathrm{eff}}(r)=4 k_{1 d}(r), \quad m_{\mathrm{eff}}(r)=\left[2 a(r)^{2} k_{1 d}(r)\right]^{-1} .
$$

Eq. 59 compares directly with the $1 \mathrm{~d}$ time-independent Schrodinger's equation,

$$
-\frac{\hbar^{2}}{2 m} \frac{d^{2}}{d r^{2}} \Psi(r)+V(r) \Psi(r)=E \Psi(r)
$$

whose local wave vector / decay rate of the wavefunction $\Psi$ is given by WKB analysis as [16 20,

$$
p= \begin{cases} \pm i \sqrt{2 m(V(r)-E)}, & \text { if } E<V(r) \\ \pm \sqrt{2 m(E-V(r))}, & \text { if } E>V(r) .\end{cases}
$$

However, Eq. 59, compared with Eq. 61, has a flipped sign in front of the double derivative term, corresponding to momentum transformation $p \rightarrow i p$. The local wave vector / decay rate of a phonon of the cone is thus given by,

$$
p= \begin{cases}\left. \pm \sqrt{2 m_{\mathrm{eff}}(r)\left(V_{\mathrm{eff}}(r)-\Omega\right.}\right), & \text { if } \Omega<V_{\mathrm{eff}}(r) \\ \pm i \sqrt{2 m_{\mathrm{eff}}(r)\left(\Omega-V_{\mathrm{eff}}(r)\right)}, & \text { if } \Omega>V_{\mathrm{eff}}(r) .\end{cases}
$$

Since $m_{\mathrm{eff}}(r)>0$ is always positive, the turning point $r^{*}$ for an eigenmode with eigenfrequency $\Omega$ is given by,

$$
\Omega=V_{\text {eff }}\left(r^{*}\right)=4 k_{1 d}\left(r^{*}\right) .
$$

The WKB domain boundary for the phonons of a cone is thus,

$$
\Omega=6 \frac{\varepsilon}{a\left(r^{*}\right)^{2}}\left[152\left(\frac{a_{0}}{a\left(r^{*}\right)}\right)^{12}-80\left(\frac{a_{0}}{a\left(r^{*}\right)}\right)^{6}\right] .
$$

Eq. 65 is exactly equivalent to the band edge criterion in Eq. (53), which again becomes Eq. (41) in the limit of small cone angle.

\section{CONCLUSION}

We demonstrate the intriguing effects smooth variations in lattice parameters can have on a discrete ordered system. In particular, we show that a two-dimensional sheet of interacting particles with cylindrical boundary conditions collapses into a truncated cone under gravitational pressure. We find that the cone shape, namely its apex angle $\theta$, controls level crossings at long wavelengths as well as the quasi-localization of normal modes at short wavelengths, with localization domains modulated by the inhomogeneous density profile. In the regime of small cone angles $\theta$, we predict the boundaries of these domains as a function of $\theta$ in Eq. 41 .

The system studied in this paper can be realized in experiments, for example, by a two-dimensional colloidal crystal (possibly in a liquid solvent) with colloids adsorbed onto the surface of a cone [12, 29] at the appropriate cone angle, given by Eq. 20 as a function of colloid's effective mass in the solvent. In this work, we have examined inertial dynamics $\frac{m}{2} \dot{u}^{2}$ for concreteness, but other types of (possibly overdamped) dynamics can also be studied by similar methods.

Similar localization phenomena may be found in other types of inhomogeneous lattices in both two and three dimensions. In particular, it will be interesting to explore the analogue of Eq. 41 in conformal lattices, e.g. flux line lattices under magnetic field gradients [3, 4, where the inhomogeneity can act not only through local dilation or contraction but also through local rotation. It is also widely known that density-mismatched bulk colloidal crystals in three dimensions exhibit smaller lattice constants at the bottom [3032, as the colloids further below become increasingly more crushed by the weight of the colloids and solvent above. It would be interesting to investigate the normal modes of these systems and see whether eigenfunction localization domains again appear at short wavelengths. Finally, in systems where the membrane is free-standing and out-of-plane fluctuations are important 33, it may be worthwhile to investigate how in-plane inhomogeneity affects the flexural phonons.

\section{ACKNOWLEDGMENTS}

We are grateful for helpful conversations with F. Spaepen and H. Zhou. G.H.Z. acknowledges support by the National Science Foundation Graduate Research Fellowship under Grant No. DGE1745303. This work was also supported by the NSF through the Harvard Materials Science and En- 
gineering Center, via Grant No. DMR-2011754, as well as by Grant No. DMR-1608501.

\section{Appendix A: Dislocation pileups}

The dynamical Hamiltonian for a one-dimensional dislocation pileup embedded in a two-dimensional host crystal is given by [14,

$$
\begin{aligned}
\mathcal{H}\left[\left\{x_{n}\right\}\right]= & \sum_{n=1}^{N} \frac{m}{2} \dot{x}_{n}^{2}+\sum_{n=1}^{N} b\left(x_{n}\right) \sigma_{0} U\left(x_{n}\right) \\
& -\frac{Y}{8 \pi} \sum_{n \neq m} b\left(x_{n}\right) b\left(x_{m}\right) \ln \left|x_{n}-x_{m}\right|,
\end{aligned}
$$

where $Y$ is the $2 \mathrm{~d}$ Young's modulus controlling the repulsive pair potential, $b(x)= \pm b$ is the Burgers vector, $m$ is the effective mass of the dislocation, and $N$ is the total number of dislocations in the pileup. In the second term, $\sigma_{0} U(x)=\int_{-L / 2}^{x} d x^{\prime} \sigma\left(x^{\prime}\right)$ comes from the PeachKoehler force due to the applied shear stress $\sigma(x)$, where $\sigma_{0}$ measures the strength of the shear stress and $U(x)$, with dimensions of length, is the spatial profile of the potential experienced by the dislocations due to the shear stress.

\section{Numerical determination of dislocation sites}

The pileups in Fig. 1 $1 \mathrm{a}$ and $\mathrm{b}$ of the main text, referred to respectively as the double pileup and the semicircle pileup, each experience the following forms of shear stress,

$$
\begin{aligned}
\sigma_{\mathrm{D}}(x) & =\sigma_{0} \\
\sigma_{\mathrm{SC}}(x) & =\sigma_{0} \frac{x}{L / 2},
\end{aligned}
$$

where $L$ is the length of the pileup, and the average dislocation charge densities are [14, 15, as plotted in Fig. 8.

$$
\begin{gathered}
n_{\mathrm{D}}(x)=\frac{4 \sigma_{0}}{Y b} \frac{x}{\sqrt{\left(\frac{L}{2}\right)^{2}-x^{2}}} \\
n_{\mathrm{SC}}(x)=\frac{4 \sigma_{0}}{Y b} \sqrt{1-\left(\frac{x}{L / 2}\right)^{2}} .
\end{gathered}
$$

Note that for the double pileup, the sign of the defect charge changes across the origin. Additionally, although $n_{D}(x)$ is singular near $x= \pm L / 2$, these defect densities vary smoothly elsewhere.

We now extract the equilibrium lattice positions numerically from the charge densities in Eqs. A4 A5). Since the absolute value of both distributions are symmetric about the pileup centers, we compute the dislocation sites on the right half of the dislocation pileup (positive $x$ axis) and reflect across the vertical axis at $x=0$ (see Fig. 8).

On writing the dislocation positions as,

$$
x_{i}=x_{i-1}+\frac{1}{2}\left[D\left(x_{i-1}\right)+D\left(x_{i}\right)\right]
$$

where $D\left(x_{i}\right)=\left|n\left(x_{i}\right)\right|^{-1}$ is the lattice constant at the position $x_{i}$ of the $i$-th dislocation, and rescaling spatially by half

(a)

(b)
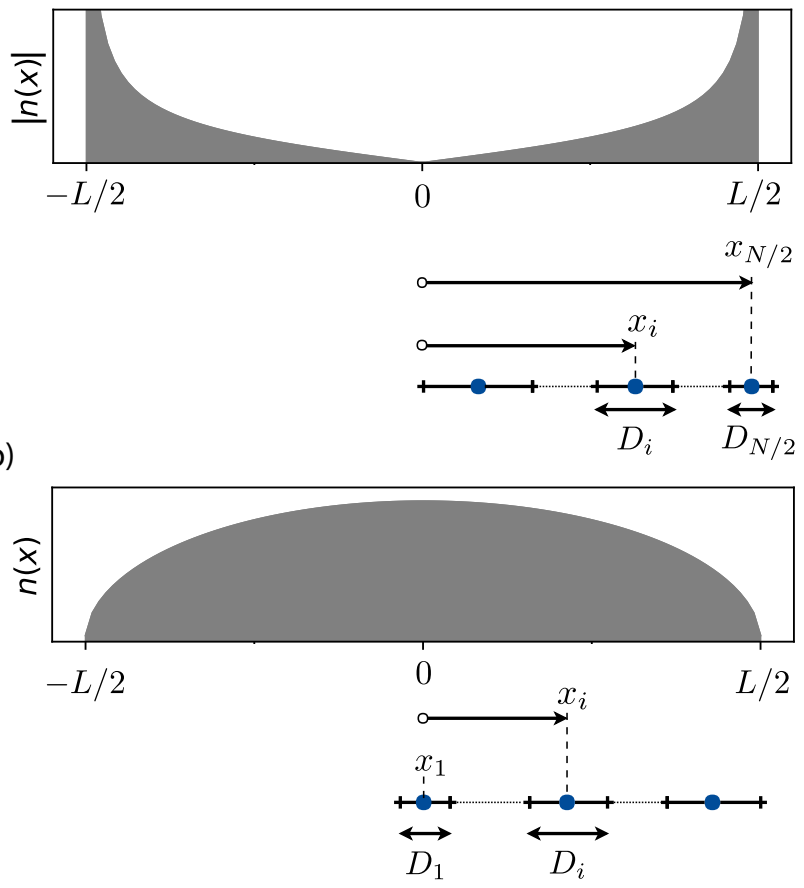

FIG. 8. Schematic illustrating the numerical determination of dislocation positions for the double pileup (a) and the semicircle pileup (b). Gray shadings plot the absolute value of the dislocation charge densities $n_{\mathrm{D}}(x)$ and $n_{\mathrm{SC}}(x)$ in Eqs. A4 A5. $x_{i}$ indicates the position of the $i$-th dislocation site from the origin, and $a_{i}$ indicates the lattice constant corresponding to the $i$-th dislocation site.

of the total length of the dislocation pileup, we have

$$
\bar{x}_{i}=\bar{x}_{i-1}+\frac{1}{2}\left[\bar{D}\left(\bar{x}_{i-1}\right)+\bar{D}\left(\bar{x}_{i}\right)\right]
$$

where $\bar{x}=x /(L / 2), \bar{D}=D /(L / 2)$. Since the dislocation density vanishes at the center for the double pileup and at the edge for the semicircle pileup, we solve for the dislocation positions iteratively starting from the center for the semicircle pileup and the edge for the double pileup, where the charge densities are finite. Upon using Eqs. A4 A5 and defining $f_{i} \equiv \bar{x}_{i}+\frac{1}{2} \bar{D}\left(\bar{x}_{i}\right)$ and $g_{i} \equiv \bar{x}_{i}-\frac{1}{2} \bar{D}\left(\bar{x}_{i}\right)$, the position $\bar{x}_{i}$ of the $i$-th dislocation can be computed from the position of the $(i-1)$-th (or $(i+1)$-th) dislocation by numerically solving for the root of these quartic equations, starting from their respective boundary conditions, 


$$
\begin{gathered}
\bar{x}_{i, D}^{4}-2 g\left(\bar{x}_{i+1, D}\right) \bar{x}_{i, D}^{3}+\left[\left(\frac{Y b}{4 L \sigma_{0}}\right)^{2}+g\left(\bar{x}_{i+1, D}\right)\right] \bar{x}_{i, D}^{2}-\left(\frac{Y b}{4 L \sigma_{0}}\right)^{2}=0, \quad \bar{x}_{N / 2, D}=1 \\
\bar{x}_{i, S C}^{4}-2 f_{i-1} \bar{x}_{i, S C}^{3}-\left(1-f\left(\bar{x}_{i-1, S C}\right)^{2}\right) \bar{x}_{i}^{2}-2 f\left(\bar{x}_{i-1, S C}\right) \bar{x}_{i}-f\left(\bar{x}_{i-1, S C}\right)^{2}-\frac{Y b}{8 L \sigma_{0}}=0, \quad \bar{x}_{1, S C}=0 .
\end{gathered}
$$

\section{Dynamical matrix and real space eigenfunctions}

On decomposing the dislocation position as $x_{n}=R_{n}+u_{n}$ (where $R_{n}$ is the equilibrium location and $u_{n}$ is the displacement from the equilibrium lattice site), and assuming the displacements to be small relative to the lattice spacing at low temperatures, we expand the Hamiltonian up to quadratic order in $u_{n}$ as,

$$
\mathcal{H}\left[\left\{u_{n}\right\}\right]=\sum_{n=1}^{N} \frac{m}{2} \dot{u}_{n}^{2}+\sum_{n, m} M_{n m} u_{n} u_{m},
$$

where the diagonal $M_{n, n}$ and off-diagonal $M_{n, m \neq n}$ matrix elements for the double pileup are given by,

$$
\begin{aligned}
M_{n, n}^{D} & =\frac{\sigma_{0} b}{N} \sum_{m \neq n} \frac{\operatorname{sign}\left(R_{n} R_{m}\right)}{\left(R_{n}-R_{m}\right)^{2}} \\
M_{n, m \neq n}^{D} & =-\frac{\sigma_{0} b}{N} \frac{\operatorname{sign}\left(R_{n} R_{m}\right)}{\left(R_{n}-R_{m}\right)^{2}},
\end{aligned}
$$

and those of the semicircle pileup by,

$$
\begin{aligned}
M_{n, n}^{S C} & =\sigma_{0} b\left(1+\frac{1}{N} \sum_{m \neq n} \frac{1}{\left(R_{n}-R_{m}\right)^{2}}\right) \\
M_{n, m \neq n}^{S C} & =-\frac{\sigma_{0} b}{N} \frac{1}{\left(R_{n}-R_{m}\right)^{2}},
\end{aligned}
$$

where the pileup lengths have been normalized such that $x \in(-1,1)$. Normalization conditions can be found in Table I of Ref. [15]. Upon parameterizing $u_{n}(t)=u_{n} e^{-i \omega t}$, we obtain the equations of motion as,

$$
\Omega u_{n}=\sum_{m} M_{n m} u_{m}
$$

where $\Omega \equiv m \omega^{2}$ is the eigenenergy.

As mentioned above, to obtain the matrix elements in Eqs. A12 A15, we compute the equilibrium dislocation positions $\left\{R_{n}\right\}$ by placing a dislocation at the center of the pileup $x=0$ and using Eqs. A4 and A5 to solve iteratively for the positions of its neighboring dislocations until the edge is reached. We then numerically compute the normal modes by solving the following eigenproblem,

$$
\mathbf{M u}^{(\alpha)}=\Omega_{\alpha} \mathbf{u}^{(\alpha)},
$$

where $\mathbf{M}$ is the dynamical matrix with elements $M_{n, m}$, and $\Omega_{\alpha} \equiv m \omega_{\alpha}^{2}$ and $\mathbf{u}^{(\alpha)}$ are the eigenenergies and eigenfunctions of the $\alpha$-th eigenmode.

\section{Phonon spectrum and band edge}

The full dispersion relation for a uniform one-dimensional pileup is given by [15],

$$
\begin{aligned}
\Omega(q) & =\frac{1}{D^{2}} \frac{Y b^{2}}{4 \pi} \sum_{n=1}^{\infty} \frac{1-\cos (n q D)}{n^{2}} \\
& =\frac{1}{D^{2}} \frac{Y b^{2}}{8 \pi}\left\{\frac{\pi^{2}}{3}-\left[\operatorname{Li}_{2}\left(e^{-i q D}\right)+\operatorname{Li}_{2}\left(e^{i q D}\right)\right]\right\}
\end{aligned}
$$

where $\operatorname{Li}_{n}(z)$ is the polylogarithmic function (or Jonquière's function) [34, $D$ is the the average dislocation spacing, $Y$ is the Young's modulus of the $2 \mathrm{~d}$ host crystal, and $b$ is the magnitude of the Burgers vector. The band edge, which occurs at the boundary of the first Brillouin zone $q=\pi / D$, is then obtained from Eq. (1) according to Eq. A18.

We show the evolution of the localization domains for the double pileup and the semicircle pileup in Fig. 11. According to Eqs. (2), A4, and (A5), the localization domains $\left(x_{\alpha}^{-}, x_{\alpha}^{+}\right)$for these pileups satisfy,

$$
\Omega_{\alpha}=\frac{\pi \sigma_{0}^{2}}{Y} \times \begin{cases}\frac{x_{\alpha}^{2}}{\left(\frac{L}{2}\right)^{2}-x_{\alpha}^{2}}, & \text { Double pileup } \\ 1-\left(\frac{x_{\alpha}}{L / 2}\right)^{2}, & \text { Semicircle }\end{cases}
$$

Note that $Y$ and $\sigma_{0}$ have units of $E / L^{2}$ in $1 \mathrm{~d}$, so the dimensions on both sides of the equation match. As shown in Fig. 1 the band edge shown in Eq. A20 agrees with the numerical eigenfunctions. As the energy of the excitation mode increases, the eigenfunction becomes increasingly more localized towards the densest parts of the lattice. For the semicircle pileup, this corresponds to the middle of the pileup, while for the double pileup, this is the region near the pileup edges.

Appendix B: 1d chain of balls and springs under gravity

An instructive toy model is a one-dimensional chain of balls of mass $m$, connected by springs with stiffness $k_{s}$, subject to gravity on a plane inclined by angle $\theta$ with respect to the ground. Although that the gravitational force causes the density of the masses to be inhomogeneous along the inclined plane, the equations of motion and thus the phonons remain identical to that of the homogeneous lattice without the effects of gravity. Thus, for this purely harmonic problem, all effects of the lattice inhomogeneity can be redefined away.

The energy that leads to phonon dynamics in this system 
(a)

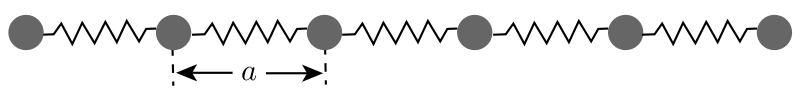

(b)

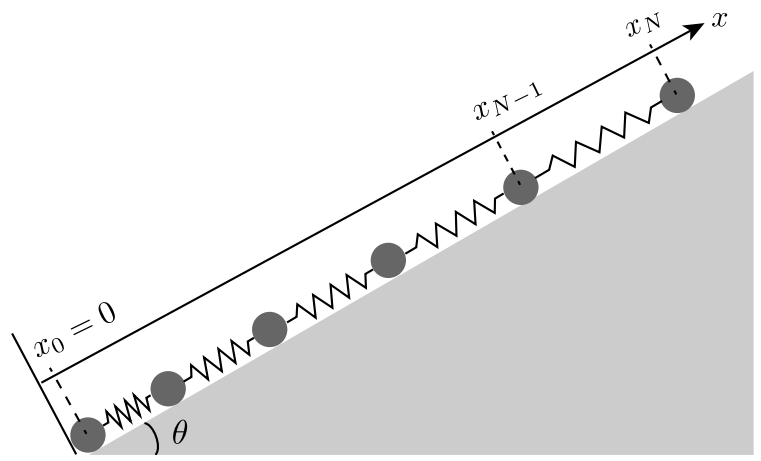

FIG. 9. Schematic for 1d problem of springs and masses on a flat surface (a) and on an inclined plane of angle $\theta$ (b). $a$ is the equilibrium distance between two neighboring balls on a flat plane. $x_{n}$ denotes the position of the $n$-th ball along the inclined plane from the bottommost ball at $x_{0}=0$.

is

$$
H\left[\left\{x_{n}\right\}\right]=\sum_{n=1}^{N}\left[\frac{m}{2} \dot{x}_{n}^{2}+\frac{1}{2} k_{s}\left(x_{n+1}-x_{n}-a\right)^{2}-m^{\prime} g x_{n}\right],
$$

where $m^{\prime}=m \sin \theta, g$ is the gravitational acceleration, and $x_{n}$ is the position (along the inclined plane) of the $n$-th ball. The lowest point of the inclined plane is met by a wall such that the first ball (indexed by $n=0$ ) has fixed position centered at $x_{0}=0$ (see Fig. 9). Upon writing the new equilibrium position as $x_{n}^{*}=n a+u_{n}^{*}$, minimizing the energy, and applying the following boundary conditions (zero displacement at $n=0$ and zero stress at $n=N$ ),

$$
u^{*}(n=0)=0,\left.\quad \frac{\partial u^{*}(n)}{\partial n}\right|_{N}=0 .
$$

we obtain the equilibrium lattice positions $\left\{x_{n}^{*}\right\}$ as,

$$
x^{*}(n)=n a+\frac{m^{\prime} g}{2 k_{s}}\left[n^{2}-2 N n\right] .
$$

Upon decomposing the mass position in Eq. (B1) as $x_{n}=x_{n}^{*}+u_{n}$, where $u_{n}$ is the displacement away from the equilibrium position $x_{n}^{*}$, and noting that terms linear in displacement vanish due to force balance, we are left with,

$$
\begin{aligned}
H\left[\left\{x_{n}\right\}\right]= & \sum_{n} \frac{m}{2} \dot{u}_{n}^{2}+\sum_{n} \frac{k_{s}}{2}\left(u_{n+1}^{2}+u_{n}^{2}-2 u_{n+1} u_{n}\right) \\
& -\sum_{n} m^{\prime} g x_{n}^{*} .
\end{aligned}
$$

Since the gravitational term in Eq. (B4) only contributes a constant shift to the energy, it does not appear in the equations of motions,

$$
-m \ddot{u}_{n}=k_{s}\left(2 u_{n}-u_{n+1}-u_{n-1}\right)
$$

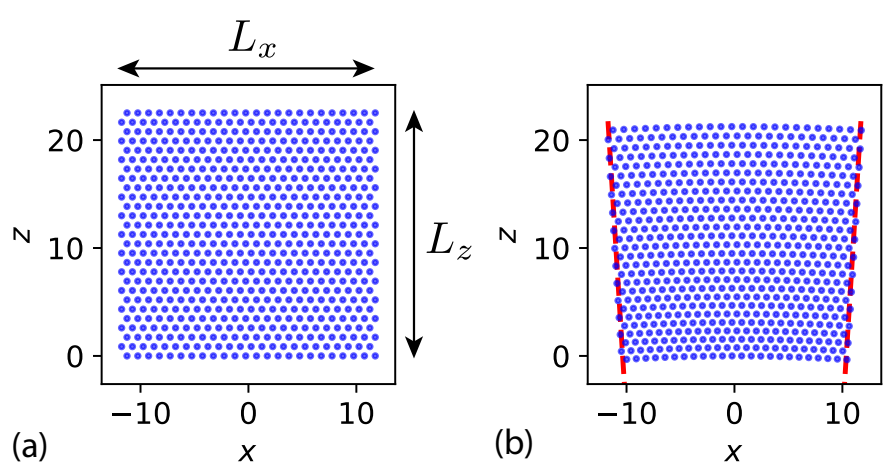

FIG. 10. (a): The unfrustrated ground state configuration of $N$ particles interacting via the Lennard-Jones potential in the flat plane is the triangular lattice with lattice constant $a_{0}$, height $L_{z}$, and width $L_{x}$. (b): When experiencing an isotropic gravitational pressure, the flat sheet becomes geometrically frustrated, curving the upper and lower boundaries and rendering solutions from simple linear elasticity theory invalid. As discussed in the main text, this frustration can be avoided by wrapping the top and bottom boundaries around to form a truncated cone.

which are identical to the equations of motion for a homogeneous chain of balls and springs. The phonon spectrum, upon assuming wave-like solutions $u_{n}=\frac{1}{\sqrt{N}} e^{i q n a} e^{i \omega t}$, is also unchanged,

$$
\omega^{2}(q)=4 \frac{k_{s}}{m} \sin ^{2}\left(\frac{q a}{2}\right) .
$$

Note that the band edge at $q=\pi / a$ is independent of the lattice spacing $a$, so the system does not exhibit the quasilocalization phenomena described in the main body of this paper. In summary, the inhomogeneity in this toy problem does not affect the phonon spectrum, because it does not appear in the interaction strengths. However, if the stiffness $k_{s}$ of the spring connecting two masses were to depend on the distance between them, then $k_{s} \rightarrow k_{s}\left(x_{n}^{*}\right)$ in the presence of inhomogeneity and that could change the equations of motions in interesting ways.

\section{Appendix C: Elastic deformation of a flat elastic sheet subjected to gravitational pressure in the plane of the sheet}

We examine a $2 \mathrm{~d}$ sheet in the flat plane with uniform elastic coupling constants under gravitational pressure using continuum elasticity theory, and show that the gravitational potential frustrates the ground state when the particles are constrained to remain on a flat plane parallel to the gravitational force. In contrast, as shown in the main text, when we allow the sheet of particles to escape into the third dimension by imposing cylindrical boundary conditions, the frustration can be gauged away, i.e. eliminated by a change of variables.

The free energy of a two-dimensional sheet in the $x$ - $z$ plane under an isotropic pressure that increases with decreasing vertical coordinate $z$ is given by,

$$
F=\frac{1}{2} \int d^{2} r\left(2 \mu u_{i k}^{2}+\lambda u_{i i}^{2}+2 \alpha_{0}\left(L_{z}-z\right) u_{i i}\right),
$$

where $\delta p=\alpha_{0}(L-z)$ is an isotropic $z$-dependent pressure, 
$u_{i j}=\frac{1}{2}\left(\frac{\partial u_{i}}{\partial r_{j}}+\frac{\partial u_{j}}{\partial r_{i}}\right)$ is the strain tensor, $\mu$ and $\lambda$ are the first and second $2 \mathrm{~d}$ Lamé coefficients. Here $\alpha_{0}$ is proportional to the gravitational constant $g$ (see Eq. (34)) and $\vec{u}(\vec{r})$ is the displacement of the sheet from its $\alpha_{0}=0$ equilibrium configuration at position $\vec{r}$. The non-deformed $\left(\alpha_{0}=0\right)$ sheet extends from $\left[0, L_{z}\right]$ along $z$ and from $\left[-L_{x} / 2, L_{x} / 2\right]$ along $x$ (Fig. 10a). The natural boundary conditions for this problem are anchoring the flat bottom edge of the sheet at the base, which requires that the vertical displacements $u_{z}$ vanish at $z=0$ and the stresses vanish at $z=L_{z}$.

We now find the set of strains $\bar{u}_{i j}$ that minimize Eq. (C1). First, since the gravitational term is isotropic and does not depend on $u_{z x}$, we immediately have

$$
\bar{u}_{z x}=0 .
$$

Next, upon setting the derivatives of the free energy $F$ with respect to $u_{z z}$ and $u_{x x}$ to zero as,

$$
\begin{aligned}
& 0=(2 \mu+\lambda) \bar{u}_{z z}(r)+\bar{u}_{x x}(r)+\alpha_{0}\left(L_{z}-z\right) \\
& 0=(2 \mu+\lambda) \bar{u}_{x x}(r)+\bar{u}_{z z}(r)+\alpha_{0}\left(L_{z}-z\right)
\end{aligned}
$$

we obtain

$$
\bar{u}_{z z}=\bar{u}_{x x}=\frac{\alpha_{0}\left(z-L_{z}\right)}{2 B},
$$

where $B=\mu+\lambda$ is the 2 d bulk modulus of the sheet.

Since $\bar{u}_{z z}=\bar{u}_{x x}=0$ at $z=L_{z}$, the stresses $\sigma_{z z}$ and $\sigma_{x x}$ also vanish there, so the free boundary condition at $z=L_{z}$ is satisfied. Upon directly integrating Eq. (C5), we obtain,

$$
\begin{aligned}
& \bar{u}_{z}=\frac{\alpha_{0}}{2 B}\left(\frac{z^{2}}{2}-L_{z} z\right)+\phi(x) \\
& \bar{u}_{x}=\frac{\alpha_{0}}{2 B}\left(z-L_{z}\right) x+\xi(z) .
\end{aligned}
$$

To determine $\phi(x)$ and $\xi(z)$, we use Eq. $\mathrm{C} 2$,

$$
\bar{u}_{z x}=0=\frac{d \phi(x)}{d x}+\frac{\alpha_{0}}{2 B} x+\frac{d \xi(z)}{d z}
$$

which leads to,

$$
\begin{aligned}
\xi(z) & =c \\
\phi(x) & =-\frac{\alpha_{0}}{2 B} \frac{x^{2}}{2}+d,
\end{aligned}
$$

where $c$ and $d$ are constants, and Eqs. $(\mathrm{C} 6)$ and (C7) become,

$$
\begin{aligned}
& \bar{u}_{z}=\frac{\alpha_{0}}{2 B}\left(\frac{z^{2}}{2}-L_{z} z-\frac{x^{2}}{2}\right)+d \\
& \bar{u}_{x}=\frac{\alpha_{0}}{2 B}\left(z-L_{z}\right) x+c .
\end{aligned}
$$

However, we immediately see that the fixed displacement boundary condition at the bottom edge of a planar sheet (as would be the case if it rested on a flat surface) cannot be satisfied, as there is no choice of $c$ and $d$ in Eqs. (C11) and (C12) such that $\bar{u}_{z}(x, z=0)=\bar{u}_{x}(x, z=0)=0$ for all $x \in\left[-L_{x} / 2, L_{x} / 2\right]$.

As an approximation, we can set $c=d=0$ in Eqs. (C11) and (C12) such that only the $(x, z)=(0,0)$ point has zero displacement. Fig. $10 \mathrm{p}$ then plots the triangular lattice sites displaced according to

$$
\begin{aligned}
& \bar{u}_{z}=\frac{\alpha_{0}}{2 B}\left(\frac{z^{2}}{2}-L_{z} z-\frac{x^{2}}{2}\right) \\
& \bar{u}_{x}=\frac{\alpha_{0}}{2 B}\left(z-L_{z}\right) x .
\end{aligned}
$$

The curl of the $2 \mathrm{~d}$ displacement field $\left(u_{x}, u_{z}\right)$ shows that the bond angles $\vartheta$ between nearest neighbors on the lattice are in fact rotating as a function $x$,

$$
\vartheta(x)=\frac{1}{2}\left(\frac{\partial u_{z}}{\partial x}-\frac{\partial u_{x}}{\partial z}\right)=-\frac{\alpha_{0}}{2 B} x .
$$

Since Eqs. C13 and (C14) cannot satisfy the bottom boundary conditions, the elasticity solutions are not valid near the bottom of the sheet. This problem also arises in three dimensions for rods deforming under a gravitational field paralle to the rod axis, where planar boundary conditions at the bottom render linear elastic solutions invalid near the lower end of the rod 22. Thus, for flat $2 \mathrm{~d}$ sheets in the flat $x-z$ plane, there is no smooth deformation that can minimize the free energy in Eq. (C1). In other words, we cannot "gauge away" the effect of gravitational pressure in the $2 \mathrm{~d}$ plane. In this situation, the extra strain energy for this wedge-shaped sheet might possibly be reduced by introducing defects, such as a vertical grain boundary at $x=0$ [35, 36]. However, we show in the main text that, remarkably, this geometric frustration can be removed entirely by wrapping the horizontal direction $x$ around to form a cylinder and thus allowing the sheet to curve into the third dimension.

\section{Appendix D: Displacements under hydrostatic compression in cone coordinates}

In this section, we derive the displacements in terms of cone coordinates $r$ (Eqs. 12, 13p) using the displacements in the Cartesian frame $(x, z)$. First, we show that the inner edge of an annulus on the polar plane corresponds to the bottom edge of our deformed sheet in the small cone angle limit. Next, we calculate the azimuthal displacement $\bar{u}_{\phi}$ using the polar cone angle and the circumference of the bottom edge of the truncated cone. Finally, upon requiring that the compression must be isotropic along both the azimuthal and axial directions, we extract the longitudinal displacement $\bar{u}_{r}$.

Recall that the displacements in the flat plane under hydrostatic compression are given by Eqs. (10 11) in the main text as,

$$
\bar{u}_{z}=\frac{\alpha_{0}}{2 B}\left(\frac{z^{2}}{2}-L_{z} z-\frac{x^{2}}{2}\right), \quad \bar{u}_{x}=\frac{\alpha_{0}}{2 B}\left(z-L_{z}\right) x,(\mathrm{D} 1)
$$

and the polar cone angle in the small angle limit is given by Eq. 20) as,

$$
\Phi=\frac{\alpha_{0}}{2 B} L_{x}+O\left(\left(\frac{\alpha_{0}}{2 B} L_{z}\right)^{2}\right) .
$$

Note that this polar cone angle vanishes when the gravitational pressure $\sim \alpha_{0} \rightarrow 0$ and when the material becomes incompressible $B=\mu+\lambda \rightarrow 0$.

Upon letting $r_{1}$ denote the inner radius of the annulus (the polar projection of a truncated cone, see Fig. 11), the inner 


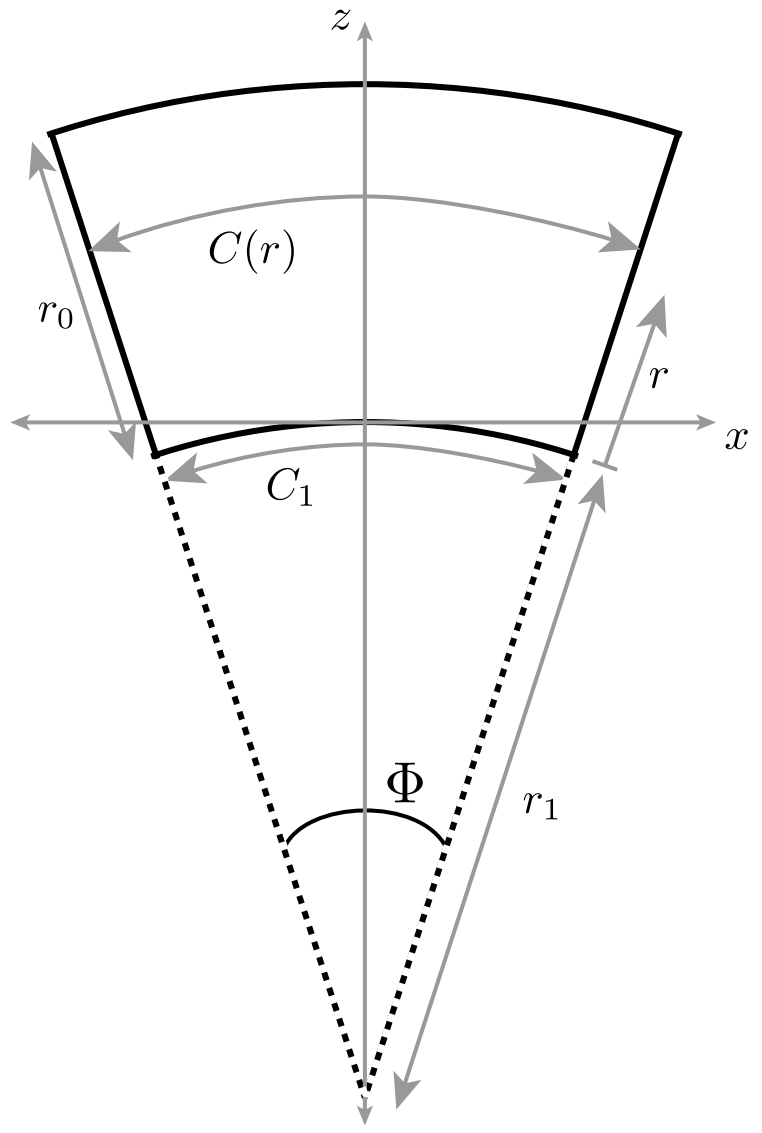

FIG. 11. Schematic illustrating the quantities mentioned in text. The solid black lines bound a $2 \mathrm{~d}$ planar crystal deformed in the $(x, z)$-plane by hydrostatic compression. Although the boundary conditions at the bottom can only be satisfied at the single point $(x, z)=(0,0)$ in the plane, this problem vanishes when we wrap this crystal around to form a small-angle truncated cone with polar angle $\Phi$. Here, $r$ is the distance up the flanks of the cone from its base.

annular rim is given by,

$$
x^{2}+\left(z+r_{1}\right)^{2}=r_{1}^{2}
$$

from which it follows that

$$
\begin{aligned}
x^{2} & =-2 z r_{1}\left(1+\frac{z}{2 r_{1}}\right) \\
& \approx-2 z r_{1},
\end{aligned}
$$

where for small cone angles, $z \ll r_{1}$, so the second term can be neglected to lowest order. We calculate $r_{1}$ specifying the tip of the cone from

$$
\Phi r_{1}=C_{1},
$$

where $C_{1}$ is the circumference of the cross-section of the cone at $r=0$ (see Fig. 11). Upon using Eqs. (D1), $C_{1}$ is given approximately by,

$$
\begin{aligned}
\left(\frac{C_{1}}{2}\right)^{2} & \approx\left(\frac{L_{x}}{2}+\bar{u}_{x}\left(x=L_{x} / 2, z=0\right)\right)^{2}+\bar{u}_{z}\left(x=L_{x} / 2, z=0\right)^{2} \\
& =\left(\frac{L_{x}}{2}\right)^{2}\left\{1-2 \frac{\alpha_{0}}{2 B} L_{z}+O\left[\left(\frac{\alpha_{0}}{2 B} L_{z}\right)^{2},\left(\frac{\alpha_{0}}{2 B} L_{x}\right)^{2}\right]\right\},
\end{aligned}
$$

which, to linear order in the cone angle, gives

$$
C_{1} \approx L_{x}\left(1-\frac{\alpha_{0}}{2 B} L_{z}\right) .
$$

Upon substituting Eq. (D7) into Eq. (D5) and using Eq. (D2), we then obtain

$$
r_{1}=\frac{\left(1-\frac{\alpha_{0}}{2 B} L_{z}\right)}{\frac{\alpha_{0}}{2 B}} .
$$

On inserting Eq. (D8) into Eq. (D4), we have

$$
\begin{aligned}
z & =-\frac{x^{2}}{2 r_{1}} \\
& =-\frac{x^{2}}{2} \frac{\frac{\alpha_{0}}{2 B}}{\left(1-\frac{\alpha_{0}}{2 B} L_{z}\right)} \\
& \approx-\frac{x^{2}}{2} \frac{\alpha_{0}}{2 B}+O\left(L_{x}\left(\frac{\alpha_{0} L_{x}}{2 B}\right)\left(\frac{\alpha_{0} L_{z}}{2 B}\right)\right),
\end{aligned}
$$

so Eq. (D9) is indeed equivalent to $\bar{u}_{z}(x, z=0)$ in Eq. (D1),

$$
\bar{u}_{z}(x, z=0)=-\frac{x^{2}}{2} \frac{\alpha_{0}}{2 B} .
$$

In the small cone angle limit where $r_{1} \gg r$ and $r_{1} \gg z$, we approximate the circumference $C(r)$ as a function of $r$ and the polar cone angle $\Phi$ as (see Fig. 11),

$$
C(r)=\Phi\left(r_{1}+r\right)=C_{1}+\Phi r=L_{x}\left[1+\frac{\alpha_{0}}{2 B}\left(r-L_{z}\right)\right] .
$$

We can then solve for the radial displacement $\bar{u}_{R}(r)$ as,

$$
2 \pi \bar{u}_{R}(r)=C(r)-L_{x}=\frac{\alpha_{0}}{2 B}\left(r-L_{z}\right) L_{x}
$$

which leads to

$$
\bar{u}_{R}(r)=\frac{1}{2 \pi} \frac{\alpha_{0}}{2 B}\left(r-L_{z}\right) L_{x} .
$$

Note that the radial displacement becomes more negative as one moves down the flanks of the cone by decreasing $r$. The radial displacement can be written as the usual azimuthal displacement $\bar{u}_{\phi}(r, \phi)$ around the axis of the cone on its tangent plane as,

$$
\bar{u}_{\phi}(r, \phi)=\phi \bar{u}_{R}(r) .
$$

To obtain the other component of the displacement field, we note that since the gravitational pressure is isotropic, the compression factor has to be the same along both the axial $(r)$ and azimuthal $(\phi)$ directions:

$$
\frac{d \bar{u}_{r}(R)}{d r}=\frac{d \bar{u}_{\phi}(r, \phi)}{d\left(L_{x} \phi / 2 \pi\right)}=\frac{\alpha_{0}}{2 B}\left(r-L_{z}\right) .
$$

Upon integrating and imposing cylindrical boundary conditions, the axial displacement $\bar{u}_{r}$ is given by

$$
\bar{u}_{r}(r)=\frac{\alpha_{0}}{2 B}\left(\frac{r^{2}}{2}-L_{z} r\right) .
$$

Using the fact that $L_{z}$ is well approximated by the longitudinal cone length $r_{0}$ in Eqs. (D14) and (D16) to linear order in the cone angle, we obtain Eqs. 12 13) in the main text. 


\section{Appendix E: Force balance for particles with nonlinear interaction potentials}

In this section, we show that under a gravitational potential, the equilibrium displacements of a lattice with elastic coefficients $\mu(r), \lambda(r)$ that depend on the local lattice spacing is the same as that of a lattice with constant elastic coefficients $\mu, \lambda$, provided that the gravitational pressure is sufficiently week. This latter condition corresponds exactly to the small cone angle limit.

To examine the nonlinear feedback between an inhomogeneous lattice and elastic constants that depend on the local lattice spacing, we write the local Lamé coefficients $\lambda(\vec{r})$ and $\mu(\vec{r})$ in terms of the strain tensor. We first write the $x$-th component of the lattice spacing $a_{x, n}$ of the $n$-th particle as,

$$
a_{x, n}=a_{0}+\left(\bar{u}_{x, n+1_{x}}-\bar{u}_{x, n}\right) \approx a_{0}\left(1+\bar{u}_{x x}\right),
$$

where $a_{0}$ is the lattice constant of the undeformed sheet $\left(\alpha_{0}=0\right)$. Here, $\bar{u}_{x, n}$ is the $x$-th component of the displacement of the $n$-th particle from its position in the unpressurized lattice, and $n+1_{x}$ denotes the index of its neighbor in the $\hat{e}_{x}$ direction. A similar expression follows for $a_{y, n}$.

To reduce notational clutter, we will show the calculation explicitly for interaction potentials of the power law form $V(\vec{r})=A /|\vec{r}|^{t}$. The results can then be transcribed to the
Lennard-Jones potential, which is a sum of two power law terms with $t=6$ and 12. Upon using Eqs. 39 and C5. the space-dependent elastic coupling coefficients for LennardJones particles on a cone may be written as,

$$
\begin{aligned}
& \lambda(\vec{r})=\frac{\lambda_{0}}{\left(1+\frac{\alpha_{0}}{B_{0}}\left(z-L_{z}\right)\right)^{t+2}} \\
& \mu(\vec{r})=\frac{\mu_{0}}{\left(1+\frac{\alpha_{0}}{B_{0}}\left(z-L_{z}\right)\right)^{t+2}}
\end{aligned}
$$

where $B_{0}=\lambda_{0}+\mu_{0}$ and $\mu_{0}, \lambda_{0}$ are the elastic constants of the undeformed lattice.

Having determined the form of the nonlinear elastic coupling coefficients $\lambda(\vec{r})$ and $\mu(\vec{r})$, we want to solve for the equilibrium positions $\left\{R_{n}\right\}$ of the particles under gravitational pressure by minimizing the following free energy,

$$
F=\frac{1}{2} \int d^{2} r\left(2 \mu(\vec{r}) u_{i j}^{2}+\lambda(\vec{r}) u_{i i}^{2}+2 \alpha_{0}\left(L_{z}-z\right) u_{i i}\right)
$$

Taking derivatives of $F$ with respect to the components of the strain tensor $u_{i j}$ yields, upon using Eq. E1 for the local lattice spacing,

$$
\begin{aligned}
& 0=(2 \mu(\vec{r})+\lambda(\vec{r})) \bar{u}_{x x}+\lambda(\vec{r}) \bar{u}_{z z}+\alpha_{0}\left(L_{z}-z\right)-\frac{(2 \mu(\vec{r})+\lambda(\vec{r}))}{2} \frac{t+2}{2} \frac{\bar{u}_{x x}^{2}+\bar{u}_{z z}^{2}}{1+\bar{u}_{x x}}-\frac{\lambda(\vec{r})}{2} \frac{t+2}{2} \frac{\left(\bar{u}_{z z}+\bar{u}_{x x}\right)^{2}}{1+\bar{u}_{z z}} \\
& 0=(2 \mu(\vec{r})+\lambda(\vec{r})) \bar{u}_{z z}+\lambda(\vec{r}) \bar{u}_{x x}+\alpha_{0}\left(L_{z}-z\right)-\frac{(2 \mu(\vec{r})+\lambda(\vec{r}))}{2} \frac{t+2}{2} \frac{\bar{u}_{x x}^{2}+\bar{u}_{z z}^{2}}{1+\bar{u}_{z z}}-\frac{\lambda(\vec{r})}{2} \frac{t+2}{2} \frac{\left(\bar{u}_{z z}+\bar{u}_{x x}\right)^{2}}{1+\bar{u}_{x x}}
\end{aligned}
$$

Upon defining the following quantity,

$$
\gamma(z) \equiv \frac{\alpha_{0}\left(L_{z}-z\right)}{2 B_{0}}=\theta \frac{z-L_{z}}{L_{x}}
$$

we can rewrite Eq. (E5) as,

$$
\begin{aligned}
& 0=\frac{2 \mu(\vec{r})+\lambda(\vec{r})}{B_{0}} \bar{u}_{x x}+\frac{\lambda(\vec{r})}{B_{0}} \bar{u}_{z z}+\gamma(z)-\frac{(2 \mu(\vec{r})+\lambda(\vec{r}))}{2 B_{0}} \frac{t+2}{2} \frac{\bar{u}_{x x}^{2}+\bar{u}_{z z}^{2}}{1+\bar{u}_{x x}}-\frac{\lambda(\vec{r})}{2 B_{0}} \frac{t+2}{2} \frac{\left(\bar{u}_{z z}+\bar{u}_{x x}\right)^{2}}{1+\bar{u}_{z z}} \\
& 0=\frac{2 \mu(\vec{r})+\lambda(\vec{r})}{B_{0}} \bar{u}_{z z}+\frac{\lambda(\vec{r})}{B_{0}} \bar{u}_{x x}+\gamma(z)-\frac{(2 \mu(\vec{r})+\lambda(\vec{r}))}{2 B_{0}} \frac{t+2}{2} \frac{\bar{u}_{x x}^{2}+\bar{u}_{z z}^{2}}{1+\bar{u}_{z z}}-\frac{\lambda(\vec{r})}{2 B_{0}} \frac{t+2}{2} \frac{\left(\bar{u}_{z z}+\bar{u}_{x x}\right)^{2}}{1+\bar{u}_{x x}}
\end{aligned}
$$

When the perturbation is small, the strains $\bar{u}_{x x}$ and $\bar{u}_{z z}$ are on the order of $\gamma(z)$,

$$
\left|\bar{u}_{z z}\right|=\left|\bar{u}_{x x}\right| \sim|\gamma(z)| \leq\left|\theta \frac{L_{z}}{L_{x}}\right| \ll 1,
$$

and we can eliminate the higher order quadratic terms $\sim \bar{u}_{i i}^{2}$ in Eq. E7 and retrieve the linear approximation displayed in Eqs. (C5):

$$
\bar{u}_{z z}=\bar{u}_{x x}=\frac{\alpha_{0}\left(z-L_{z}\right)}{2 B_{0}}+O\left(\gamma^{2}\right)
$$

The condition in Eq. (E8), for an approximately square sheet $L_{x} \sim L_{z}$, precisely corresponds to the small angle condition in Eq. 8). Thus, when the gravitational pressure is sufficiently weak, nonlinear effects can be neglected and the equilibrium deformation of particles with power law pair interactions is identical to that of the two-dimensional sheet with uniform elasticity constants. Since the Lennard-Jones potential is the sum of two power law terms, the previous statements also hold for the system studied in the main text, where the particles interact via the LJ potential in Eq. (6). 


\section{Appendix F: Phonon spectrum derivations}

\section{Free energy functional of the cone}

Upon letting $u_{i k}=\bar{u}_{i k}+\delta u_{i k}$, the free energy in Eq. (9) becomes,

$$
\begin{aligned}
F= & \frac{1}{2} \int d^{2} r\left[2 \mu\left(\bar{u}_{i k}^{2}+2 \bar{u}_{i k} \delta u_{i k}+\delta u_{i k}^{2}\right)\right. \\
& \left.+\lambda\left(\bar{u}_{i i}^{2}+2 \bar{u}_{j j} \delta u_{i i}+\delta u_{i i}^{2}\right)+2 \alpha_{0}\left(L_{z}-z\right)\left(\bar{u}_{i i}+\delta u_{i i}\right)\right] .
\end{aligned}
$$

Upon grouping the terms linear and quadratic in $\delta u_{i k}$,

$$
\begin{aligned}
F= & F_{0}+\frac{1}{2} \int d^{2} r 2 \delta u_{i k}\left[2 \mu \bar{u}_{i k}+\lambda \bar{u}_{j j} \delta_{i k}+\alpha_{0}\left(L_{z}-z\right) \delta_{i k}\right] \\
& +\frac{1}{2} \int d^{2} r\left[2 \mu \delta u_{i k}^{2}+\lambda \delta u_{i i}^{2}\right]
\end{aligned}
$$

where $F_{0}$ is the energy of the equilibrium configuration without displacements,

$$
F_{0}=\frac{1}{2} \int d^{2} r\left(2 \mu \bar{u}_{i k}^{2}+\lambda \bar{u}_{i i}^{2}+2 \alpha_{0}\left(L_{z}-z\right) \bar{u}_{i i}\right),
$$

we see that the coefficient of the term linear in $\delta u_{i k}$ vanishes according to Eq. C3. Hence, the change in free energy due to displacements away from equilibrium becomes,

$$
F=F_{0}+\delta F=F_{0}+\frac{1}{2} \int d^{2} r\left[2 \mu \delta u_{i k}^{2}+\lambda \delta u_{i i}^{2}\right],
$$

and $\delta F\left(\delta u_{i k}\right)$ as a function of the new displacements takes the same form as that of the free energy of the unpressurized uniform $2 \mathrm{~d}$ sheet. Thus, for a system with constant elastic coefficients $\mu$ and $\lambda$, the phonon spectrum of the deformed lattice would be the same as the undeformed lattice. However, for particles interacting via the Lennard-Jones potential, the inhomogeneous equilibrium configuration causes the elastic constants to become non-uniform $\mu, \lambda \rightarrow \mu(\vec{r}), \lambda(\vec{r})$. As shown in Sec. IV] this has significant effects on the normal modes.

\section{Results for power law interaction potentials}

In this section, we derive several key quantities for particles interacting via a power law potential of the following form,

$$
V_{\mathrm{int}}\left(\vec{x}_{n}, \vec{x}_{m}\right)=\frac{A}{\left|\vec{x}_{n}-\vec{x}_{m}\right| t}
$$

where $A$ is some constant coefficient and $t$ is the exponent of the power law. Since the Lennard-Jones potential is the sum of two power law terms, the results are readily applied to the system in the main text.

Note that when $t=3$, Eq. (F5) captures the physics of experimentally relevant systems such as magnetic colloids, whose pair potential takes the form [37,

$$
U(r)=\frac{\mu_{0}(\chi B)^{2}}{8 \pi} \frac{1}{r^{3}}
$$

where $r$ is the distance between two colloids, $\chi$ is the magnetic susceptibility, $B$ is the magnitude of the external magnetic field, and $\mu_{0}$ is the permeability constant. However, note that particles experiencing a purely repulsive interaction potential such as Eq. (F6) requires an external potential to prevent them from flying apart. In contrast, since the Lennard-Jones potential has both a repulsive component $\sim 1 / r^{12}$ and an attractive component $\sim 1 / r^{6}$, a separate external potential is not needed to confine the particles.

\section{a. Dispersion relation}

For interaction potentials of the form $V(r)=A /|r|^{t}(t>$ $0)$, the matrix element in Eq. 43 becomes

$$
\Pi_{i j}(\Delta \vec{R})=A\left[t(t+2) \frac{\Delta R_{i} \Delta R_{j}}{|\Delta \vec{R}|^{t+4}}-t \frac{\delta_{i j}}{|\Delta \vec{R}|^{t+2}}\right] .
$$

To reduce notational clutter in the upcoming calculations, we abbreviate Eq. (F7) as,

$$
\Pi_{i j}(\vec{R})=\gamma \frac{R_{i} R_{j}}{|\vec{R}|^{t+4}}-\rho \frac{\delta_{i j}}{|\vec{R}|^{t+2}},
$$

where,

$$
\gamma \equiv A t(t+2), \quad \rho \equiv A t .
$$

Upon following the procedure delineated in Sec. IV C, summing over the nearest neighbor lattice vectors $\left\{\vec{n}_{\alpha}\right\}$, and using the following trigonometric identities,

$$
\begin{aligned}
\cos (a+b)+\cos (a-b) & =2 \cos (a) \cos (b) \\
\cos (a+b)-\cos (a-b) & =-2 \sin (a) \sin (b) \\
\cos (2 a) & =2 \cos ^{2}(a)-1,
\end{aligned}
$$

the dynamical matrix in momentum space $D_{i j}(\vec{q})$ (see e.g. Eq. (51) ) takes the following form,

$$
D(\vec{q})=\frac{4}{a^{t+2}}\left(\begin{array}{cc}
(\gamma-\rho)\left(1-c_{x}^{2}\right)+\left(\frac{\gamma}{4}-\rho\right)\left(1-c_{x} c_{z}\right) & \gamma \frac{\sqrt{3}}{4} s_{x} s_{z} \\
\gamma \frac{\sqrt{3}}{4} s_{x} s_{z} & -\rho\left(1-c_{x}^{2}\right)+\left(\frac{3 \gamma}{4}-\rho\right)\left(1-c_{x} c_{z}\right)
\end{array}\right)
$$

where

$$
c_{x} \equiv \cos \left(q_{x} \frac{a}{2}\right), \quad c_{z} \equiv \cos \left(q_{z} a \frac{\sqrt{3}}{2}\right)
$$

$$
s_{x} \equiv \sin \left(q_{x} \frac{a}{2}\right), \quad s_{z} \equiv \sin \left(q_{z} a \frac{\sqrt{3}}{2}\right) .
$$

The eigenenergies near the Brillouin zone center (see Fig. 5), e.g. along the $\Gamma-K$ line $\left(q_{x} a=q a \ll 1, q_{z} a=0\right)$ and 
along the $\Gamma-M$ line $\left(q_{z} a=q a \ll 1, q_{x} a=0\right)$, are given by

$$
\begin{aligned}
& \Omega_{T}=(q a)^{2} \frac{3}{8} \frac{\gamma-4 \rho}{a^{t+2}}=(q a)^{2} \frac{3}{8} \frac{t^{2}-2 t}{a^{t+2}} \\
& \Omega_{L}=(q a)^{2} \frac{9}{8} \frac{\gamma-\frac{4}{3} \rho}{a^{t+2}}=(q a)^{2} \frac{9}{8} \frac{t^{2}-\frac{2}{3} t}{a^{t+2}} .
\end{aligned}
$$

Note that here, the longitudinal eigenenergy is not equal to 3 times the transverse eigenenergy. This change arises because the usual Cauchy condition [23] is modified by the requirement of an external pressure to prevent the particles, which are interacting here via a purely repulsive potential, from flying apart.

The band edge at point $M$ in the Brillouin zone on the longitudinal dispersion curve is given by

$$
\Omega_{\text {edge }}(A, t)=\frac{6}{a^{t+2}}\left(\gamma-\frac{4}{3} \rho\right)=\frac{6 A}{a^{t+2}}\left(t^{2}+\frac{2}{3} t\right) .
$$

where we have used the values of $\gamma$ and $\rho$ from Eq. (F9). It is now straightforward to calculate the band edge for the LJ potential in Eq. (6) as

$$
\Omega_{\text {edge }}=\Omega\left(A=\varepsilon a_{0}^{12}, t=12\right)-\Omega\left(A=2 \varepsilon a_{0}^{6}, t=6\right)(
$$

which gives Eq. 53 in the main text.

\section{b. Effect of next nearest neighbors}

Because the Lennard-Jones potential falls off rapidly with distance, corrections to the spectrum due to further neighbors are expected to be small. Here, we examine the effects of incorporating next nearest neighbor (NNN) interactions on the spectrum, in particular the dispersion near the zone center and the band edge.

For power law interactions of the form $V(r)=A /|r|^{t}$, the next nearest neighbor (see dashed lines in Fig. 12) contribution to the dynamical matrix is,

$$
D^{N N N}(\vec{q})=\frac{4}{(\sqrt{3} a)^{t+2}}\left(\begin{array}{cc}
-\rho\left(1-\bar{c}_{z}^{2}\right)+\left(\frac{3 \gamma}{4}-\rho\right)\left(1-\bar{c}_{x} \bar{c}_{z}\right) & \gamma \frac{\sqrt{3}}{4} \bar{s}_{x} \bar{s}_{z} \\
\gamma \frac{\sqrt{3}}{4} \bar{s}_{x} \bar{s}_{z} & (\gamma-\rho)\left(1-\bar{c}_{z}^{2}\right)+\left(\frac{\gamma}{4}-\rho\right)\left(1-\bar{c}_{x} \bar{c}_{z}\right)
\end{array}\right)
$$

where,

$$
\begin{array}{ll}
\bar{c}_{x} \equiv \cos \left(3 q_{x} a / 2\right), & \bar{c}_{z} \equiv \cos \left(q_{z} a \sqrt{3} / 2\right), \\
\bar{s}_{x} \equiv \sin \left(3 q_{x} a / 2\right), & \bar{s}_{z} \equiv \sin \left(q_{z} a \sqrt{3} / 2\right) .
\end{array}
$$

Near the zone center (e.g. $q_{z}=0, q_{x}=q \approx 0$ ), the contribution to the dynamical matrix from next nearest neighbors is equal to the nearest neighbor contribution scaled by an overall constant,

$$
D^{N N N}(q \approx 0)=\frac{1}{3^{t / 2}} D^{N N}(q \approx 0) .
$$

Thus, the addition of next nearest neighbor interactions scales the slopes of both dispersion branches near the zone center by the same constant factor and doesn't alter the Poisson ratio.

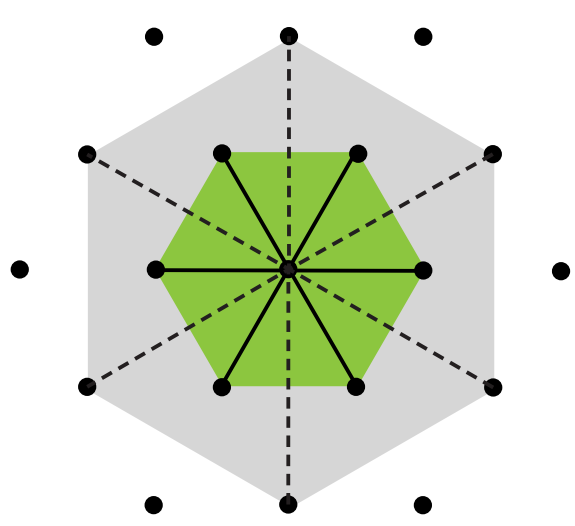

FIG. 12. Schematic indicating the nearest neighbor (solid lines) and next nearest neighbor (dashed lines) interactions in real space on a triangular lattice.

The shift in band edge due to NNN interactions is,

$$
\Delta \Omega_{\text {edge }}^{N N N}(A, t)=\frac{2 A}{(\sqrt{3} a)^{t+2}}\left(t^{2}-2 t\right) .
$$

For a uniform lattice $\left(a=a_{0}\right)$ of particles with LJ interactions (Eq. (6)), the NNN modification becomes,

$$
\Delta \Omega_{\text {edge }}^{N N N}=-\frac{784}{729} \frac{\varepsilon}{a_{0}^{2}} \approx-1.0754 \frac{\varepsilon}{a_{0}^{2}},
$$

which is indeed very small compared to the nearest neighbor contribution in Eq. (52). We thus neglect the effect of next nearest neighbors in our calculations.

\section{Real space dynamical matrix $M$}

The eigenvalue problem in Eq. (45) can be solved by diagonalizing the dynamical matrix $\mathbf{M}$ given by,

$$
\mathbf{M}=\left[\begin{array}{ll}
\tilde{\Pi}_{11} & \tilde{\Pi}_{12} \\
\tilde{\Pi}_{21} & \tilde{\Pi}_{22}
\end{array}\right]
$$

For fixed displacement boundary conditions at the bottom, each $\tilde{\boldsymbol{\Pi}}_{\mathbf{i j}}$ is a block matrix of size $\left(N-N_{\text {row }}\right) \times\left(N-N_{\text {row }}\right)$, where $N_{\text {row }} \equiv L_{x} / a_{0}$ is the number of particles in one horizontal row (the triangular lattice is oriented such that the top and bottom edges are flat). Diagonalizing $\mathbf{M}$ then gives us a total of $2\left(N-N_{\text {row }}\right)$ normal modes. The off-diagonal elements $\tilde{\Pi}_{i j}^{(n \neq m)}$ are given by

$$
\tilde{\Pi}_{\mathbf{i j}}^{(n m)}=\Pi_{i j}\left(\vec{R}_{n}-\vec{R}_{m}\right)
$$


where $\Pi_{i j}(\vec{R})$ is given in Eq. (44), $\vec{R}_{n}$ is the equilibrium position of the $n$-th particle, and $n$ and $m$ only include the $N-N_{\text {row }}$ particle indices that exclude those in the bottom fixed row. The diagonal elements are given by,

$$
\tilde{\Pi}_{\mathbf{i j}}^{(n n)}=-\sum_{m^{\prime} \neq n} \Pi_{i j}\left(\vec{R}_{n}-\vec{R}_{m^{\prime}}\right)
$$

where $m^{\prime}$ is summed over all $N$ particle indices, including those in the bottom row. Finally, the eigenvector $\mathbf{u}^{(\alpha)}$ takes the form,

$$
\begin{aligned}
\mathbf{u}^{T^{(\alpha)}}= & {\left[u_{1}^{(\alpha)}\left(\vec{R}_{1}\right), \cdots, u_{1}^{(\alpha)}\left(R_{N-N_{\text {row }}}\right),\right.} \\
& \left.u_{2}^{(\alpha)}\left(\vec{R}_{1}\right), \cdots, u_{2}^{(\alpha)}\left(R_{N-N_{\text {row }}}\right)\right] .
\end{aligned}
$$

[20] G. Green et al., Transactions of the Cambridge Philosophical Society 6, 457 (1838).

11] M. Kac, The American Mathematical Monthly 73, 1 (1966). Physical review letters 68, 3555 (1992).

[3] R. M. Menezes, E. Sardella, L. R. Cabral, and C. C. de Souza Silva, Journal of Physics: Condensed Matter 31, 175402 (2019)

[4] F. C. Silva, R. M. Menezes, L. R. Cabral, and C. C. de Souza Silva, Journal of Physics: Condensed Matter 32, 505401 (2020).

[5] H. Ansell, A. Tomlinson, and N. Wilkin, Scientific reports 10, 1 (2020).

[6] H. Thomas, G. Morfill, V. Demmel, J. Goree, B. Feuerbacher, and D. Möhlmann, Physical Review Letters 73, 652 (1994).

[7] A. Mughal and M. Moore, Physical Review E 76, 011606 (2007).

[8] H. M. Thomas and G. E. Morfill, Nature 379, 806 (1996).

[9] W. Drenckhan, D. Weaire, and S. Cox, European journal of physics 25, 429 (2004).

[10] M. Mielenz, J. Brox, S. Kahra, G. Leschhorn, M. Albert, T. Schätz, H. Landa, and B. Reznik, Physical review letters 110, 133004 (2013).

[11] V. Soni, L. R. Gómez, and W. T. Irvine, Physical Review X 8, 011039 (2018).

[12] J. Sun, N. Tanjeem, and V. Manoharan, Bulletin of the American Physical Society 65 (2020).

[13] T. Rehman, N. Tanjeem, and V. Manoharan, in APS March Meeting Abstracts, Vol. 2019 (2019) pp. G70-060.

[14] J. P. Hirth and J. Lothe, Theory of dislocations (New York: McGraw-Hill, 1968).

[15] G. H. Zhang and D. R. Nelson, Physical Review E 103, 022139 (2021).

[16] G. Wentzel, Zeitschrift für Physik 38, 518 (1926).

[17] H. A. Kramers, Zeitschrift für Physik 39, 828 (1926).

[18] L. Brillouin, CR Acad. Sci 183, 24 (1926).

[19] H. Jeffreys, Proceedings of the London Mathematical Society 2, 428 (1925).
[21] N. Ashcroft and N. Mermin, Acad. Press, NY 33 (1978).

[22] L. Landau, L. Pitaevskii, A. Kosevich, and E. Lifshitz, Theory of Elasticity: Volume 7, v. 7 (Elsevier Science, 2012).

[23] J. H. Weiner, Statistical mechanics of elasticity (Courier Corporation, 2012).

[24] D. S. Fisher, B. Halperin, and R. Morf, Physical Review B 20, 4692 (1979).

[25] D. S. Fisher, Physical Review B 22, 1190 (1980).

[26] M. L. Mehta, Random matrices (Elsevier, 2004).

[27] E. P. Wigner, SIAM review 9, 1 (1967).

[28] L. D. Landau and E. M. Lifshitz, Quantum mechanics: nonrelativistic theory, Vol. 3 (Elsevier, 2013).

[29] A. Plummer, J. Sun, V. Manoharan, and D. Nelson, Bulletin of the American Physical Society (2021).

[30] W. B. Russel, W. Russel, D. A. Saville, and W. R. Schowalter, Colloidal dispersions (Cambridge university press, 1991).

[31] M. Rutgers, J. Dunsmuir, J.-Z. Xue, W. Russel, and P. Chaikin, Physical Review B 53, 5043 (1996).

[32] K. Jensen, D. Pennachio, D. Recht, D. Weitz, and F. Spaepen, Soft Matter 9, 320 (2013).

[33] D. Nelson, T. Piran, and S. Weinberg, Statistical mechanics of membranes and surfaces (World Scientific, 2004).

[34] A. Erdelyi, W. Magnus, F. Oberhettinger, and F. Tricomi, New York (1953).

[35] G. H. Zhang and D. R. Nelson, Physical Review Letters 125, 215503 (2020).

[36] Q. Meng and G. M. Grason, arXiv preprint arXiv:2106.08520 (2021).

[37] U. Gasser, C. Eisenmann, G. Maret, and P. Keim, ChemPhysChem 11, 963 (2010). 